\title{
Extended Target Tracking Using a Gaussian- Mixture PHD Filter
}

Karl Granström, Christian Lundquist and Umut Orguner

\section{Linköping University Post Print}

N.B.: When citing this work, cite the original article.

C2012 IEEE. Personal use of this material is permitted. However, permission to reprint/republish this material for advertising or promotional purposes or for creating new collective works for resale or redistribution to servers or lists, or to reuse any copyrighted component of this work in other works must be obtained from the IEEE.

Karl Granström, Christian Lundquist and Umut Orguner, Extended Target Tracking Using a Gaussian-Mixture PHD Filter, 2012, IEEE Transactions on Aerospace and Electronic Systems, (48), 4, 3268-3286.

http://dx.doi.org/10.1109/TAES.2012.6324703

Postprint available at: Linköping University Electronic Press

http://urn.kb.se/resolve?urn=urn:nbn:se:liu:diva-71866 


\title{
Extended Target Tracking using a Gaussian-Mixture PHD filter
}

\author{
Karl Granström, Member, IEEE, Christian Lundquist, and Umut Orguner, Member, IEEE
}

\begin{abstract}
This paper presents a Gaussian-mixture implementation of the PHD filter for tracking extended targets. The exact filter requires processing of all possible measurement set partitions, which is generally infeasible to implement. A method is proposed for limiting the number of considered partitions and possible alternatives are discussed. The implementation is used on simulated data and in experiments with real laser data, and the advantage of the filter is illustrated. Suitable remedies are given to handle spatially close targets and target occlusion.
\end{abstract}

Index Terms-Target tracking, extended target, PHD filter, random set, Gaussian-mixture, laser sensor.

\section{INTRODUCTION}

In most multi-target tracking applications it is assumed that each target produces at most one measurement per time step. This is true for the cases when the distance between the target and the sensor is large in comparison to the target's size. In other cases however, the target size may be such that multiple resolution cells of the sensor are occupied by the target. Targets that potentially give rise to more than one measurement per time step are categorized as extended. Examples include the cases when vehicles use radar sensors to track other road-users, when ground radar stations track airplanes which are sufficiently close to the sensor, or in mobile robotics when pedestrians are tracked using laser range sensors.

Gilholm and Salmond [1] have presented an approach for tracking extended targets under the assumption that the number of received target measurements in each time step is Poisson distributed. Their algorithm was illustrated with two examples where point targets which may generate more than one measurement and objects that have a 1-D extension (infinitely thin stick of length $l$ ) are tracked. In [2] a measurement model was suggested which is an inhomogeneous Poisson point process. At each time step, a Poisson distributed random number of measurements are generated, distributed around the target. This measurement model can be understood to imply that the extended target is sufficiently far away from the sensor for its measurements to resemble a cluster of points, rather than a geometrically structured ensemble. A similar approach is taken in [3] where track-before-detect theory is used to track a point target with a 1-D extent.

The authors gratefully acknowledge the fundings from the Swedish Foundation for Strategic Research (SSF) under the project Collaborative Unmanned Aircraft Systems (CUAS), and from the Swedish Research Council under the Linnaeus Center CADICS and under the frame project grant Extended Target Tracking (621-2010-4301).

The authors are with the Department of Electrical Engineering, Division of Automatic Control, Linköping University, Linköping, SE-581 83, Sweden, e-mail: $\{$ karl,lundquist,umut $\} @$ isy.liu.se.
Baum et al have presented the random hypersurface model [4], an extended target model which has been used to track elliptic targets [5], as well as more general shapes [6]. A different approach to elliptic target modeling is the random matrix framework by Koch [7]. The target kinematical states are modeled using a Gaussian distribution, while the ellipsoidal target extension is modeled using an inverse Wishart distribution. Using random matrices to track group targets under kinematical constraints is discussed in [8]. Modifications and improvements to the Gaussian-inverse Wishart model of [7] have been suggested in [9], and the model [7] has been integrated into a Probabilistic Multi-Hypothesis Tracking (РMHT) framework in [10]. A comparison of random matrices and the random hypersurface model under single target assumption is given in [11]. Measurements of target down-range extent are used to aid track retention in [12]. Other approaches to estimating the target extensions are given in [13]-[15].

Using the rigorous finite set statistics (FISST), Mahler has pioneered the recent advances in the field of multiple target tracking with a set theoretic approach where the targets and measurements are treated as random finite sets (RFS). This type of approach allows the problem of estimating multiple targets in clutter and uncertain associations to be cast in a Bayesian filtering framework [16], which in turn results in an optimal multi-target Bayesian filter. As is the case in many nonlinear Bayesian estimation problems, the optimal multitarget Bayesian filter is infeasible to implement except for simple examples and an important contribution of FISST is to provide structured tools in the form of the statistical moments of a RFS. The first order moment of a RFS is called probability hypothesis density (PHD), and it is an intensity function defined over the state space of the targets. The so called PHD filter [16], [17] propagates in time PHDs corresponding to the set of target states as an approximation of the optimal multi-target Bayesian filter. A practical implementation of the PHD filter is provided by approximating the PHDs with Gaussian-mixtures (GM) [18] which results in the Gaussian-mixture PHD (GM-PHD) filter. In the recent work [19], Mahler presented an extension of the PHD filter to also handle extended targets of the type presented in [2].

In this paper, we present a Gaussian-mixture implementation of the PHD-filter for extended targets [19], which we call the extended target GM-PHD-filter (ET-GM-PHD). In this way, we, to some extent, give a practical extension of the series of work in [2], [18], [19]. An earlier version of this work was presented in [20] and the current, significantly improved, version includes not only much more details and extensive investigations of the methodology but also practical examples 
with real data. For space considerations, we do not repeat the derivation of the PHD-filter equations for extended targets and instead refer the reader to [19].

The document is outlined as follows. The multiple extended target tracking problem is defined in Section II. The details of the Gaussian-mixture implementation are given in Section III. For the measurement update step of the ET-GM-PHD-filter, different partitions of the set of measurements have to be considered. A measurement clustering algorithm used to reduce the combinatorially exploding number of possible measurement partitions is described in Section IV. The proposed approaches are evaluated using both simulations and experiments. The target tracking setups for these evaluations are described in Section V, the simulation results are presented in Section VI and results using data from a laser sensor are presented in Section VII. Finally, Sections VIII and IX contain conclusions and thoughts on future work.

\section{TARgEt TRACKING PROBLEM FORMULATION}

In previous work, extended targets have often been modeled as targets having a spatial extension or shape that would lead to multiple measurements, as opposed to at most a single measurement. On the other hand, the extended target tracking problem can be simplified by the assumption that the measurements originating from a target are distributed approximately around a target reference point [1] which can be e.g. the centroid or any other point depending on the extent (or the shape) of the target. Though all targets obviously have a spatial extension and shape, in the latter type of modeling, only the target reference point is important and the target extent does not need to be estimated.

The relevant target characteristics that are to be estimated form the target's state vector $\mathbf{x}$. Generally, beside the kinematic variables as position, velocity and orientation, the state vector may also contain information about the target's spatial extension. As mentioned above, when the target's state does not contain any variables related to the target extent, though the estimation is done as if the target was a point (i.e. the target reference point), the algorithms should still take care of the multiple measurements that originate from a target. Hence, in this study, we use a generalized definition of an extended target, given below, which does not depend on whether the target extent is estimated or not.

Definition 1 (Extended Target). A target which potentially gives rise to more than one measurement per time step.

In this work, to simplify the presentation, no information about the size and shape of the target is kept in the state vector $\mathbf{x}$, i.e. the target extent is not explicitly estimated. Nevertheless, it must be emphasized that this causes no loss of generality as shown by the recent work [13] where the resulting ET-GM-PHD filter is used to handle the joint estimation of size, shape and kinematic variables for rectangular and elliptical extended targets. We model both the target states to be estimated, and the measurements collected, as RFSs. The motivation behind this selection is two-fold. First, in many practical systems, although the sensor reports come with a specific measurement order, the results of the target tracking algorithms are invariant under permutations of this order. Hence, modeling the measurements as elements of a set in which the order of the elements is irrelevant makes sense. Second, this work unavoidably depends on the previous line of work [19], which is based on such a selection.

The initial GM-PHD work [18] does not provide tools for ensuring track continuity, for which some remedies are described in the literature, see e.g. [21]. However it has been shown that labels for the Gaussian components can be included into the filter in order to obtain individual target tracks, see e.g. [22]. In this work, for the sake of simplicity, labels are not used, however they can be incorporated as in [22] to provide track continuity.

We denote the unknown number of targets $N_{x, k}$, and the set of target states to be estimated at time $k$ is $\mathbf{X}_{k}=\left\{\mathbf{x}_{k}^{(i)}\right\}_{i=1}^{N_{x, k}}$. The measurement set obtained at time $k$ is $\mathbf{Z}_{k}=\left\{\mathbf{z}_{k}^{(i)}\right\}_{i=1}^{N_{z, k}}$ where $N_{z, k}$ is the number of measurements.

The dynamic evolution of each target state $\mathbf{x}_{k}^{(i)}$ in the RFS $\mathbf{X}_{k}$ is modeled using a linear Gaussian dynamical model,

$$
\mathbf{x}_{k+1}^{(i)}=F_{k} \mathbf{x}_{k}^{(i)}+G_{k} \mathbf{w}_{k}^{(i)}
$$

for $i=1, \ldots, N_{x, k}$, where $\mathbf{w}_{k}^{(i)}$ is Gaussian white noise with covariance $Q_{k}^{(i)}$. Note that each target state evolves according to the same dynamic model independent of the other targets.

The number of measurements generated by the $i$ th target at each time step is a Poisson distributed random variable with rate $\gamma\left(\mathbf{x}_{k}^{(i)}\right)$ measurements per scan, where $\gamma(\cdot)$ is a known non-negative function defined over the target state space. The probability of the $i$ th target generating at least one measurement is then given as

$$
1-e^{-\gamma\left(\mathbf{x}_{k}^{(i)}\right)} .
$$

The $i$ th target is detected with probability $p_{\mathrm{D}}\left(\mathbf{x}_{k}^{(i)}\right)$ where $p_{\mathrm{D}}(\cdot)$ is a known non-negative function defined over the target state space. This gives the effective probability of detection

$$
\left(1-e^{-\gamma\left(\mathbf{x}_{k}^{(i)}\right)}\right) p_{\mathrm{D}}\left(\mathbf{x}_{k}^{(i)}\right) .
$$

The measurements originating from the $i$ th target are assumed to be related to the target state according to a linear Gaussian model

$$
\mathbf{z}_{k}^{(j)}=H_{k} \mathbf{x}_{k}^{(i)}+\mathbf{e}_{k}^{(j)}
$$

where $\mathbf{e}_{k}^{(j)}$ is white Gaussian noise with covariance $R_{k}$. Each target is assumed to give rise to measurements independently of the other targets. We emphasize here, that in an RFS framework both the set of measurements $\mathbf{Z}_{k}$ and the set of target states $\mathbf{X}_{k}$ are unlabeled, and hence no assumptions are made regarding which target gives rise to which measurement.

The number of clutter measurements generated at each time step is a Poisson distributed random variable with rate $\beta_{F A, k}$ clutter measurements per surveillance volume per scan. Thus, if the surveillance volume is $V_{s}$, the mean number of clutter measurements is $\beta_{F A, k} V_{s}$ clutter measurements per scan. The 
spatial distribution of the clutter measurements is assumed uniform over the surveillance volume.

The aim is now to obtain an estimate of the sets of the target states $\mathbf{X}^{K}=\left\{\mathbf{X}_{k}\right\}_{k=1}^{K}$ given the sets of measurements $\mathbf{Z}^{K}=\left\{\mathbf{Z}_{k}\right\}_{k=1}^{K}$. We achieve this by propagating the predicted and updated PHDs of the set of target states $\mathbf{X}_{k}$, denoted $D_{k \mid k-1}(\cdot)$ and $D_{k \mid k}(\cdot)$, respectively, using the PHD filter presented in [19].

\section{GAUSSIAN-MiXTURE IMPLEMENTATION}

In this section, following the derivation of the GM-PHDfilter for standard single measurement targets in [18], a PHD recursion for the extended target case is described. Since the prediction update equations of the extended target PHD filter are the same as those of the standard PHD filter [19], the Gaussian mixture prediction update equations of the ET-GMPHD filter are the same as those of the standard GM-PHD filter in [18]. For this reason, here we only consider the measurement update formulas for the ET-GM-PHD filter.

The predicted PHD has the following Gaussian-mixture representation

$$
D_{k \mid k-1}(\mathbf{x})=\sum_{j=1}^{J_{k \mid k-1}} w_{k \mid k-1}^{(j)} \mathcal{N}\left(\mathbf{x} ; m_{k \mid k-1}^{(j)}, P_{k \mid k-1}^{(j)}\right)
$$

where

- $J_{k \mid k-1}$ is the predicted number of components;

- $w_{k \mid k-1}^{(j)}$ is the weight of the $j$ th component;

- $m_{k \mid k-1}^{(j)}$ and $P_{k \mid k-1}^{(j)}$ are the predicted mean and covariance of the $j$ th component;

- $\mathcal{N}(x ; m, P)$ denotes a Gaussian distribution defined over the variable $x$ with mean $m$ and covariance $P$.

The PHD measurement update equation for the extended target Poisson model of [2] was derived in [19]. The corrected PHD-intensity is given by the multiplication of the predicted PHD and a measurement pseudo-likelihood function [19] $L_{\mathbf{Z}_{k}}$,

$$
D_{k \mid k}(\mathbf{x} \mid \mathbf{Z})=L_{\mathbf{Z}_{k}}(\mathbf{x}) D_{k \mid k-1}(\mathbf{x} \mid \mathbf{Z}) .
$$

The measurement pseudo-likelihood function $L_{\mathbf{Z}_{k}}$ in (6) is defined as

$$
\begin{aligned}
L_{\mathbf{Z}_{k}}(\mathbf{x}) \triangleq & -\left(1-e^{-\gamma(\mathbf{x})}\right) p_{\mathrm{D}}(\mathbf{x})+e^{-\gamma(\mathbf{x})} p_{\mathrm{D}}(\mathbf{x}) \\
& \times \sum_{\mathrm{p} \angle \mathbf{Z}_{k}} \omega_{\mathrm{p}} \sum_{W \in \mathrm{p}} \frac{\gamma(\mathbf{x})^{|W|}}{d_{W}} \cdot \prod_{\mathbf{z}_{k} \in W} \frac{\phi_{\mathbf{z}_{k}}(\mathbf{x})}{\lambda_{k} c_{k}\left(\mathbf{z}_{k}\right)} .
\end{aligned}
$$

where

- $\lambda_{k} \triangleq \beta_{F A, k} V_{s}$ is the mean number of clutter measurements;

- $c_{k}\left(\mathbf{z}_{k}\right)=1 / V_{s}$ is the spatial distribution of the clutter over the surveillance volume;

- the notation $\mathrm{p} \angle \mathbf{Z}_{k}$ means that $\mathrm{p}$ partitions the measurement set $\mathbf{Z}_{k}$ into non-empty cells $W$;

- the quantities $\omega_{\mathrm{p}}$ and $d_{W}$ are non-negative coefficients defined for each partition $\mathrm{p}$ and cell $W$ respectively.

- $\phi_{\mathbf{z}_{k}}(\mathbf{x})=p\left(\mathbf{z}_{k} \mid \mathbf{x}\right)$ is the likelihood function for a single target generated measurement, which would be a Gaussian density in this work.
The first summation on the right hand side of (7) is taken over all partitions $\mathrm{p}$ of the measurement set $\mathbf{Z}_{k}$. The second summation is taken over all cells $W$ in the current partition p.

In order to derive the measurement update of the GM-PHDfilter, six assumptions were made in [18], which are repeated here for the sake of completeness.

Assumption 1. All of the targets evolve and generate observations independently of one another.

Assumption 2. Clutter is Poisson and independent of targetoriginated measurements.

Assumption 3. The predicted multi-target RFS is Poisson.

Assumption 4. Each target follows a linear Gaussian dynamical model, cf. (1), and the sensor has a linear Gaussian measurement model, $c f$. (4).

Assumption 5. The survival and detection probabilities are state independent, i.e. $p_{\mathrm{S}}(\mathbf{x})=p_{\mathrm{S}}$ and $p_{\mathrm{D}}(\mathbf{x})=p_{\mathrm{D}}$.

Assumption 6. The intensities of the birth and spawn RFS are Gaussian-mixtures.

In this paper we adopt all of the above assumptions except that we relax the assumption on detection probability as follows.

Assumption 7. The following approximation about the probability of detection function $p_{\mathrm{D}}(\cdot)$ holds,

$$
\begin{aligned}
& p_{\mathrm{D}}(\mathbf{x}) \mathcal{N}\left(\mathbf{x} ; m_{k \mid k-1}^{(j)}, P_{k \mid k-1}^{(j)}\right) \\
& \approx p_{\mathrm{D}}\left(m_{k \mid k-1}^{(j)}\right) \mathcal{N}\left(\mathbf{x} ; m_{k \mid k-1}^{(j)}, P_{k \mid k-1}^{(j)}\right)
\end{aligned}
$$

for all $\mathbf{x}$ and for $j=1, \ldots, J_{k \mid k-1}$.

Assumption 7 is weaker than Assumption 5 in that (8) is trivially satisfied when $p_{\mathrm{D}}(\cdot)=p_{\mathrm{D}}$, i.e. when $p_{\mathrm{D}}(\cdot)$ is constant. In general, Assumption 7 holds approximately when the function $p_{\mathrm{D}}(\cdot)$ does not vary much in the uncertainty zone of a target determined by the covariance $P_{k \mid k-1}^{(j)}$. This is true either when $p_{\mathrm{D}}(\cdot)$ is a sufficiently smooth function or when the signal to noise ratio (SNR) is high enough such that $P_{k \mid k-1}^{(j)}$ is sufficiently small. We still note here that Assumption 7 is only for the sake of simplification rather than approximation, since $p_{\mathrm{D}}(\mathbf{x})$ can always be approximated as a mixture of exponentials of quadratic functions (or equivalently as Gaussians) without losing the Gaussian-mixture structure of the corrected PHD, see [18]. This, however, would cause a multiplicative increase in the number of components in the corrected PHD, which would in turn make the algorithm need more aggressive pruning and merging operations. A similar approach to variable probability of detection has been taken in order to model the clutter notch in ground moving target indicator target tracking [23].

For the expected number of measurements from the targets, represented by $\gamma(\cdot)$, similar remarks apply and we use the following assumption. 
Assumption 8. The following approximation about $\gamma(\cdot)$ holds,

$$
\begin{aligned}
& e^{-\gamma(\mathbf{x})} \gamma^{n}(\mathbf{x}) \mathcal{N}\left(\mathbf{x} ; m_{k \mid k-1}^{(j)}, P_{k \mid k-1}^{(j)}\right) \\
& \quad \approx e^{-\gamma\left(m_{k \mid k-1}^{(j)}\right)} \gamma^{n}\left(m_{k \mid k-1}^{(j)}\right) \mathcal{N}\left(\mathbf{x} ; m_{k \mid k-1}^{(j)}, P_{k \mid k-1}^{(j)}\right)
\end{aligned}
$$

for all $\mathbf{x}, n=1,2, \ldots$ and $j=1, \ldots, J_{k \mid k-1}$.

The trivial situation $\gamma(\cdot)=\gamma$, i.e. when $\gamma(\cdot)$ is constant, is again a special case where Assumption 8 is satisfied. In general, satisfying Assumption 8 is more difficult than Assumption 7 and a Gaussian mixture assumption for $\gamma(\cdot)$ would not work due to the exponential function. Nevertheless Assumption 8 is expected to hold approximately either when $\gamma(\cdot)$ is a sufficiently smooth function or when the signal to noise ratio (SNR) is high enough such that $P_{k \mid k-1}^{(j)}$ is sufficiently small.

With the assumptions presented above, the posterior intensity at time $k$ is a Gaussian-mixture given by

$$
D_{k \mid k}(\mathbf{x})=D_{k \mid k}^{\mathrm{ND}}(\mathbf{x})+\sum_{\mathrm{p} \angle \mathbf{Z}_{k}} \sum_{W \in \mathrm{p}} D_{k \mid k}^{\mathrm{D}}(\mathbf{x}, W) .
$$

The Gaussian-mixture $D_{k \mid k}^{\mathrm{ND}}(\cdot)$, handling the no detection cases, is given by

$$
\begin{aligned}
D_{k \mid k}^{\mathrm{ND}}(\mathbf{x}) & =\sum_{j=1}^{J_{k \mid k-1}} w_{k \mid k}^{(j)} \mathcal{N}\left(\mathbf{x} ; m_{k \mid k}^{(j)}, P_{k \mid k}^{(j)}\right), \\
w_{k \mid k}^{(j)} & =\left(1-\left(1-e^{-\gamma^{(j)}}\right) p_{\mathrm{D}}^{(j)}\right) w_{k \mid k-1}^{(j)}, \\
m_{k \mid k}^{(j)} & =m_{k \mid k-1}^{(j)}, \quad P_{k \mid k}^{(j)}=P_{k \mid k-1}^{(j)} .
\end{aligned}
$$

where we used the short hand notations $\gamma^{(j)}$ and $p_{\mathrm{D}}^{(j)}$ for $\gamma\left(m_{k \mid k-1}^{(j)}\right)$ and $p_{\mathrm{D}}\left(m_{k \mid k-1}^{(j)}\right)$ respectively.

The Gaussian-mixture $D_{k \mid k}^{\mathrm{D}}(\mathbf{x}, W)$, handling the detected target cases, is given by

$$
\begin{aligned}
D_{k \mid k}^{\mathrm{D}}(\mathbf{x}, W) & =\sum_{j=1}^{J_{k \mid k-1}} w_{k \mid k}^{(j)} \mathcal{N}\left(\mathbf{x} ; m_{k \mid k}^{(j)}, P_{k \mid k}^{(j)}\right), \\
w_{k \mid k}^{(j)} & =\omega_{\mathrm{p}} \frac{\Gamma^{(j)} p_{\mathrm{D}}^{(j)}}{d_{W}} \Phi_{W}^{(j)} w_{k \mid k-1}^{(j)}, \\
\Gamma^{(j)} & =e^{-\gamma^{(j)}}\left(\gamma^{(j)}\right)^{|W|} \\
\Phi_{W}^{(j)} & =\phi_{W}^{(j)} \prod_{\mathbf{z}_{k} \in W} \frac{1}{\lambda_{k} c_{k}\left(\mathbf{z}_{k}\right)}
\end{aligned}
$$

where the product is over all measurements $\mathbf{z}_{k}$ in the cell $W$ and $|W|$ is the number of elements in $W$. The coefficient $\phi_{W}^{(j)}$ is given by

$$
\phi_{W}^{(j)}=\mathcal{N}\left(\mathbf{z}_{W} ; \mathbf{H}_{W} m_{k \mid k-1}^{(j)}, \mathbf{H}_{W} P_{k \mid k-1}^{(j)} \mathbf{H}_{W}^{\mathrm{T}}+\mathbf{R}_{W}\right)
$$

and is calculated using

$$
\begin{aligned}
\mathbf{z}_{W} & \triangleq \bigoplus_{\mathbf{z}_{k} \in W} \mathbf{z}_{k}, \quad \mathbf{H}_{W}=[\underbrace{H_{k}^{\mathrm{T}}, H_{k}^{\mathrm{T}}, \cdots, H_{k}^{\mathrm{T}}}_{|W| \text { times }}]^{\mathrm{T}}, \\
\mathbf{R}_{W} & =\operatorname{blkdiag}(\underbrace{R_{k}, R_{k}, \cdots, R_{k}}_{|W| \text { times }}) .
\end{aligned}
$$

The operation $\bigoplus$ denotes vertical vectorial concatenation. The partition weights $\omega_{\mathrm{p}}$ can be interpreted as the probability of the partition $\mathrm{p}$ being true and are calculated as

$$
\begin{aligned}
\omega_{\mathrm{p}} & =\frac{\prod_{W \in \mathrm{p}} d_{W}}{\sum_{\mathrm{p}^{\prime} \angle \mathbf{z}_{k}} \prod_{W^{\prime} \in \mathrm{p}^{\prime}} d_{W^{\prime}}}, \\
d_{W} & =\delta_{|W|, 1}+\sum_{\ell=1}^{J_{k \mid k-1}} \Gamma^{(\ell)} p_{\mathrm{D}}^{(\ell)} \Phi_{W}^{(\ell)} w_{k \mid k-1}^{(\ell)},
\end{aligned}
$$

where $\delta_{i, j}$ is the Kronecker delta. The mean and covariance of the Gaussian components are updated using the standard Kalman measurement update,

$$
\begin{aligned}
m_{k \mid k}^{(j)} & =m_{k \mid k-1}^{(j)}+\mathbf{K}_{k}^{(j)}\left(\mathbf{z}_{W}-\mathbf{H}_{W} m_{k \mid k-1}^{(j)}\right), \\
P_{k \mid k}^{(j)} & =\left(I-\mathbf{K}_{k}^{(j)} \mathbf{H}_{W}\right) P_{k \mid k-1}^{(j)}, \\
\mathbf{K}_{k}^{(j)} & =P_{k \mid k-1}^{(j)} \mathbf{H}_{W}^{\mathrm{T}}\left(\mathbf{H}_{W} P_{k \mid k-1}^{(j)} \mathbf{H}_{W}^{\mathrm{T}}+\mathbf{R}_{W}\right)^{-1} .
\end{aligned}
$$

In order to keep the number of Gaussian components at a computationally tractable level, pruning and merging is performed as in [18].

\section{Partitioning the Measurement Set}

As observed in the previous section, an integral part of extended target tracking with the PHD filter is the partitioning of the set of measurements [19]. The partitioning is important, since more than one measurement can stem from the same target. Let us exemplify ${ }^{1}$ the process of partitioning with a measurement set containing three individual measurements, $\mathbf{Z}_{k}=\left\{\mathbf{z}_{k}^{(1)}, \mathbf{z}_{k}^{(2)}, \mathbf{z}_{k}^{(3)}\right\}$. This set can be partitioned in the following different ways;

$$
\begin{aligned}
& \mathrm{p}_{1}: W_{1}^{1}=\left\{\mathbf{z}_{k}^{(1)}, \mathbf{z}_{k}^{(2)}, \mathbf{z}_{k}^{(3)}\right\}, \\
& \mathrm{p}_{2}: W_{1}^{2}=\left\{\mathbf{z}_{k}^{(1)}, \mathbf{z}_{k}^{(2)}\right\}, W_{2}^{2}=\left\{\mathbf{z}_{k}^{(3)}\right\}, \\
& \mathrm{p}_{3}: W_{1}^{3}=\left\{\mathbf{z}_{k}^{(1)}, \mathbf{z}_{k}^{(3)}\right\}, W_{2}^{3}=\left\{\mathbf{z}_{k}^{(2)}\right\}, \\
& \mathrm{p}_{4}: W_{1}^{4}=\left\{\mathbf{z}_{k}^{(2)}, \mathbf{z}_{k}^{(3)}\right\}, W_{2}^{4}=\left\{\mathbf{z}_{k}^{(1)}\right\}, \\
& \mathrm{p}_{5}: W_{1}^{5}=\left\{\mathbf{z}_{k}^{(1)}\right\}, W_{2}^{5}=\left\{\mathbf{z}_{k}^{(2)}\right\}, W_{3}^{5}=\left\{\mathbf{z}_{k}^{(3)}\right\} .
\end{aligned}
$$

Here, $\mathrm{p}_{i}$ is the $i$ th partition, and $W_{j}^{i}$ is the $j$ th cell of partition $i$. Let $\left|\mathrm{p}_{i}\right|$ denote the number of cells in the partition, and let $\left|W_{j}^{i}\right|$ denote the number of measurements in the cell. It is quickly realized that as the size of the measurement set increases, the number of possible partitions grows very large. In order to have a computationally tractable target tracking method, only a subset of all possible partitions can be considered. In order to achieve good extended target tracking results, this subset of partitions must represent the most likely ones of all possible partitions.

\footnotetext{
${ }^{1}$ This example was also utilized in [19].
} 
In Section IV-A, we propose a simple heuristic for finding this subset of partitions, which is based on the distances between the measurements. Note that our proposed method is only one instance of a vast number of other clustering algorithms found in the literature, and that other methods could have been used. Some well-known alternatives are pointed out, and compared to the proposed partitioning method, in Section IV-B. An addition to the partitioning approach to better handle targets which are spatially close is described in Section IV-C.

\section{A. Distance Partitioning}

Consider a set of measurements $\mathbf{Z}=\left\{\mathbf{z}^{(i)}\right\}_{i=1}^{N_{z}}$. Our partitioning algorithm relies on the following theorem.

Theorem 1. Let $\mathbf{d}(\cdot, \cdot)$ be a distance measure and $d_{\ell} \geq 0$ be an arbitrary distance threshold. Then there is one and only one partition in which any pair of measurements $\mathbf{z}^{(i)}, \mathbf{z}^{(j)} \in \mathbf{Z}$ that satisfy

$$
\mathbf{d}\left(\mathbf{z}^{(i)}, \mathbf{z}^{(j)}\right) \leq d_{\ell}
$$

are in the same cell.

Proof: The proof is given in Appendix A for the sake of clarity.

Given a distance measure $\mathbf{d}(\cdot, \cdot)$, the distances between each pair of measurements can be calculated as

$$
\Delta_{i j} \triangleq \mathbf{d}\left(\mathbf{z}^{(i)}, \mathbf{z}^{(j)}\right), \text { for } 1 \leq i \neq j \leq N_{z} .
$$

Theorem 1 says that there is a unique partition that leaves all pairs $(i, j)$ of measurements satisfying $\Delta_{i j} \leq d_{\ell}$ in the same cell. An example algorithm that can be used to obtain this unique partition is given in Table I. This algorithm is used to generate $N_{d}$ alternative partitions of the measurement set $\mathbf{Z}$, by selecting $N_{d}$ different thresholds

$$
\left\{d_{\ell}\right\}_{\ell=1}^{N_{d}}, d_{\ell}<d_{\ell+1}, \text { for } \ell=1, \ldots, N_{d}-1 .
$$

The alternative partitions contain fewer cells as the $d_{\ell}$ 's are increasing, and the cells typically contain more measurements.

The thresholds $\left\{d_{\ell}\right\}_{\ell=1}^{N_{d}}$ are selected from the set

$$
\mathcal{D} \triangleq\{0\} \cup\left\{\Delta_{i j} \mid 1 \leq i<j \leq N_{z}\right\}
$$

where the elements of $\mathcal{D}$ are sorted in ascending order. If one uses all of the elements in $\mathcal{D}$ to form alternative partitions, $|\mathcal{D}|=N_{z}\left(N_{z}-1\right) / 2+1$ partitions are obtained. Some partitions resulting from this selection might still turn out to be identical, and must hence be discarded so that each partition at the end is unique. Among these unique partitions, the first (corresponding to the threshold $d_{1}=0$ ) would contain $N_{z}$ cells with one measurement each. The last partition would have just one cell containing all $N_{z}$ measurements. Notice that this partitioning methodology already reduces the number of partitions tremendously.

In order to further reduce the computational load, partitions in this work are computed only for a subset of thresholds in the set $\mathcal{D}$. This subset is determined based on the statistical properties of the distances between the measurements belonging to the same target.
TABLE I

Distance PARTITIONING

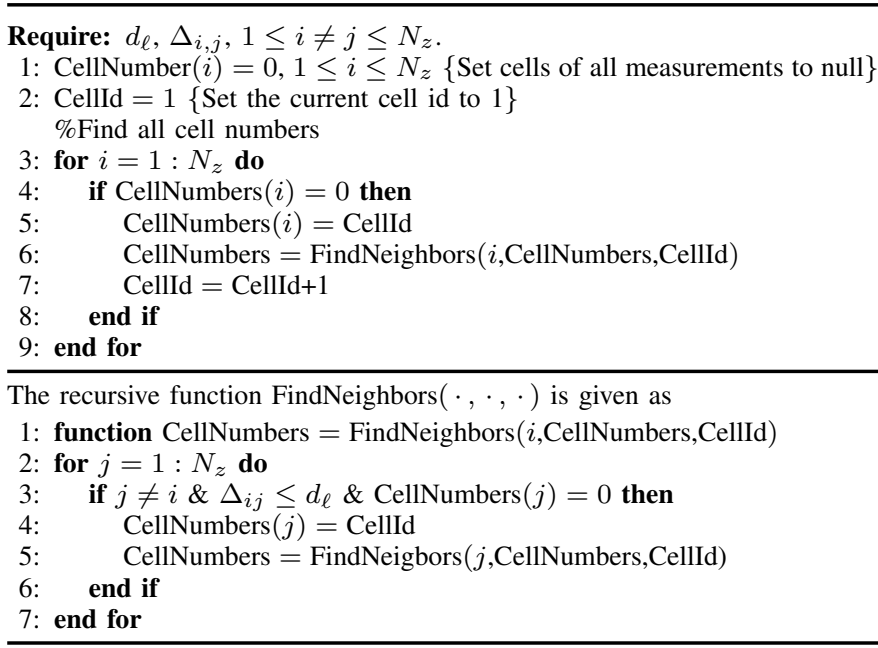

Suppose we select the distance measure $\mathbf{d}(\cdot, \cdot)$ as the Mahalanobis distance, given by

$$
\mathbf{d}_{M}\left(\mathbf{z}^{(i)}, \mathbf{z}^{(j)}\right)=\sqrt{\left(\mathbf{z}^{(i)}-\mathbf{z}^{(j)}\right)^{\mathrm{T}} R^{-1}\left(\mathbf{z}^{(i)}-\mathbf{z}^{(j)}\right)} .
$$

Then, for two target-originated measurements $\mathbf{z}^{(i)}$ and $\mathbf{z}^{(j)}$ belonging to the same target, $\mathbf{d}_{M}\left(\mathbf{z}^{(i)}, \mathbf{z}^{(j)}\right)$ is $\chi^{2}$ distributed with degrees of freedom equal to the measurement vector dimension. Using the inverse cumulative $\chi^{2}$ distribution function, denoted invchi2 $2(\cdot)$, a unitless distance threshold,

$$
\delta_{P_{\mathrm{G}}}=\operatorname{invchi} 2\left(P_{\mathrm{G}}\right),
$$

can be computed for a given probability $P_{\mathrm{G}}$. Simulations have shown that good target tracking results are achieved with partitions computed using the subset of distance thresholds in $\mathcal{D}$ satisfying the condition $\delta_{P_{\mathrm{L}}}<d_{\ell}<\delta_{P_{\mathrm{U}}}$, for lower probabilities $P_{\mathrm{L}} \leq 0.3$ and upper probabilities $P_{\mathrm{U}} \geq 0.8$.

As a simple example, if there are four targets present, each with expected number of measurements 20 , and clutter measurements are generated with $\beta_{F A} V_{s}=50$, then the mean number of measurements collected each time step would be 130. For 130 measurements, the number of all possible partitions is given by the Bell number $B_{130} \propto 10^{161}$ [24]. Using all of the thresholds in the set $\mathcal{D}, 130$ different partitions would be computed on average. Using the upper and lower probabilities $P_{\mathrm{L}}=0.3$ and $P_{\mathrm{U}}=0.8$, Monte Carlo simulations show that on average only 27 partitions are computed, representing a reduction of computational complexity several orders of magnitude.

\section{B. Alternative Partitioning Methods}

An alternative to using the proposed algorithm is to use a method which takes as input the final desired number of cells, denoted $K$, and then divides the set of measurements into $K$ cells. The most well-known example of such a method is perhaps $K$-means clustering, see e.g. the textbooks [25], [26]. In the ET-GM-PHD-filter, one needs to generate alternative partitions, corresponding to different values of $K$ between a lower and an upper threshold, denoted $K_{\mathrm{L}}$ and $K_{\mathrm{U}}$. While the 

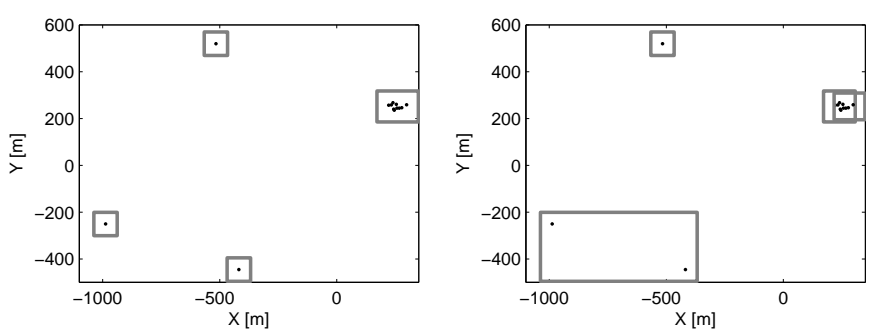

Fig. 1. Set of $N_{z, k}=13$ measurements. Left: The measurements partitioned using the suggested distance partitioning method with a distance threshold of $25 \mathrm{~m}$. Right: The measurements partitioned using $K$-means clustering with $K=4$.

values for the partitioning parameters $\delta_{P_{\mathrm{L}}}$ and $\delta_{P_{\mathrm{U}}}$ in Distance Partitioning can be chosen using some intuitive arguments as above, it is less clear how $K_{\mathrm{L}}$ and $K_{\mathrm{U}}$ should be selected. One idea is to set $K_{\mathrm{L}}=1$ and $K_{\mathrm{U}}=\left|\mathbf{Z}_{k}\right|$, which corresponds to $\delta_{P_{\mathrm{U}}}=\infty$ and $\delta_{P_{\mathrm{L}}}=0$ in Distance Partitioning. Doing so would significantly increase the computational complexity compared to Distance Partitioning, since a considerably higher number of partitions must be considered.

Another major difference between the suggested distance partitioning and $K$-means clustering is highlighted in Fig. 1, which shows a measurement set that consists of $N_{z, k}=13$ measurements, 10 of which are clustered in the northeast of the surveillance region and the other three are scattered individually. The intuitive way to cluster this set of measurements is into 4 clusters, which is achieved by Distance Partitioning using a distance threshold of about $25 \mathrm{~m}$, as shown in the left plot of Fig. 1. When there is a large number of measurements concentrated in one part of the surveillance area, as is the case in this example, $K$-means clustering tends to split those measurements into smaller cells, and merge remaining but far away measurements into large cells. This is illustrated in the right plot of Fig. 1 .

One reason behind this shortcoming of $K$-means is the initialization of the algorithm, where the initial cluster centers are chosen by uniform sampling. In order to overcome this problem, modifications to the standard $K$-means algorithm have been suggested, where initial clusters are chosen as separated as possible, see [27], [28]. This improved version of $K$-means is called $K$-means++.

In simulations, Distance Partitioning was compared to $K$ means ++ (using an implementation available online [29]). The results, see Section VI-B, show that $K$-means ++ fails to compute informative partitions much too often, except in scenarios with very low $\beta_{F A, k}$. This can be attributed to the existence of counter-intuitive local optima for the implicit cost function involved with $K$-means ++ (or $K$-means). Distance Partitioning on the other hand can handle both high and low $\beta_{F A, k}$, and always gives an intuitive and unique partitioning for a given $d_{\ell}$.

Therefore, we argue that a hierarchical method, such as the suggested Distance Partitioning, should be preferred over methods such as $K$-means. However, it is important to note here again, that regarding partitioning of the measurement set, the contribution of the current work lies mainly not in the specific method that is suggested, but rather in showing that all possible partitions can efficiently be approximated using a subset of partitions.

\section{Sub-Partitioning}

Initial results using ET-GM-PHD showed problems with underestimation of target set cardinality in situations where two or more extended targets are spatially close [20]. The reason for this is that when targets are spatially close, so are their resulting measurements. Thus, using Distance Partitioning, measurements from more than one measurement source will be included in the same cell $W$ in all partitions $\mathrm{p}$, and subsequently the ET-GM-PHD filter will interpret measurements from multiple targets as having originated from just one target. In an ideal situation, where one could consider all possible partitions of the measurement set, there would be alternative partitions which would contain the subsets of a wrongly merged cell. Such alternative partitions would dominate the output of the ET-GM-PHD filter towards the correct estimated number of targets. Since we eliminate such partitions completely using Distance Partitioning, the ET-GMPHD filter lacks the means to correct its estimated number of targets.

One remedy for this problem is to form additional partitions after performing Distance Partitioning, and to add them to the list of partitions that ET-GM-PHD filter considers at the current time step. Obviously, this should be done only when there is a risk of having merged the measurements belonging to more than one target, which can be decided based on the expected number of measurements originating from a target. We propose the following procedure for the addition of more partitions.

Suppose that we have computed a set of partitions using Distance Partitioning, e.g. with the algorithm in Table I. Then, for each generated partition $\mathrm{p}_{i}$, we calculate the maximum likelihood (ML) estimates $\hat{N}_{x}^{j}$ of the number of targets for each cell $W_{j}^{i}$. If this estimate is larger than one, we split the cell $W_{j}^{i}$ into $\hat{N}_{x}^{j}$ smaller cells, denoted

$$
\left\{W_{s}^{+}\right\}_{s=1}^{\hat{N}_{x}^{j}}
$$

We then add a new partition, consisting of the new cells along with the other cells in $\mathrm{p}_{i}$, to the list of partitions obtained by Distance Partitioning.

We illustrate the Sub-Partition algorithm in Table II, where the splitting operation on a cell is shown by a function

$$
\operatorname{split}\left(\hat{N}_{x}^{j}, W_{j}^{i}\right) .
$$

We give the details for obtaining the ML estimate $\hat{N}_{x}^{j}$ and choosing the function $\operatorname{split}(\cdot, \cdot)$ in the subsections below.

1) Computing $\hat{N}_{x}^{j}$ : For this operation, we assume that the function $\gamma(\cdot)$ determining the expected number measurements generated by a target is constant, i.e. $\gamma(\cdot)=\gamma$. Each target generates measurements independently of the other targets, and the number of generated measurements by each target is distributed with the Poisson distribution, Pois $(\cdot, \gamma)$. The 
TABLE II

SUB-PARTITION

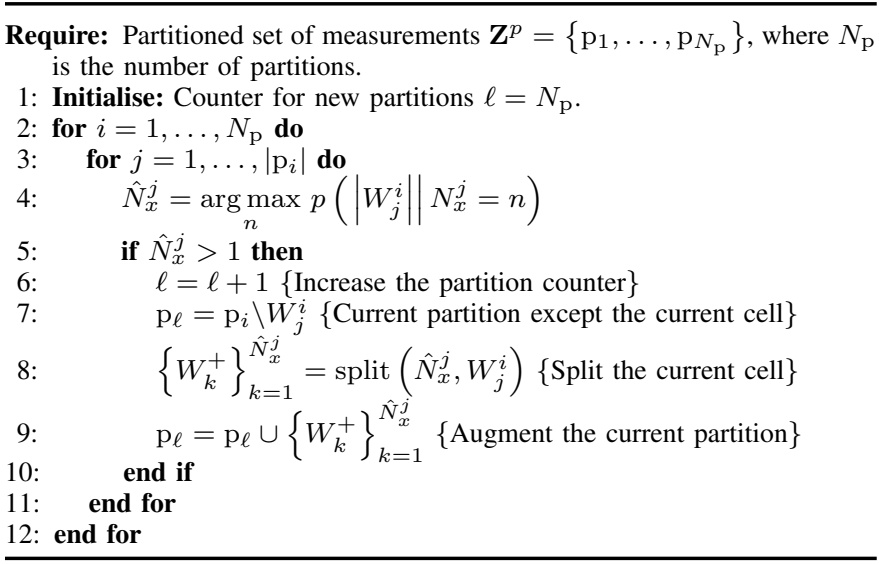

likelihood function for the number of targets corresponding to a cell $W_{j}^{i}$ is

$$
p\left(\left|W_{j}^{i}\right| \mid N_{x}^{j}=n\right)=\operatorname{Pois}\left(\left|W_{j}^{i}\right|, \gamma n\right) .
$$

Here, we assume that the volume covered by a cell is sufficiently small such that the number of false alarms in the cell is negligible, i.e. there are no false alarms in $W_{j}^{i}$. The ML estimate $\hat{N}_{x}^{j}$ can now be calculated as

$$
\hat{N}_{x}^{j}=\underset{n}{\arg \max } p\left(\mid W_{j}^{i} \| N_{x}^{j}=n\right) .
$$

Note that other alternatives can be found for calculating the estimates of $N_{x}^{j}$, e.g. utilizing specific knowledge about the target tracking setup, however both simulations and experiments have shown that the above suggested method works well.

2) The split $(\cdot, \cdot)$ function: An important part of the SubPartition function in Table II is the function split $(\cdot, \cdot)$, which is used to divide the measurements in a cell into smaller cells. In both simulations and experiments, we have used $K$-means clustering to split the measurements in the cell, results shows that this works well. However note that other methods to split the measurements are possible.

Remark 1 (Limitations of Sub-Partition). Notice that the SubPartition algorithm given in this section can be interpreted to be only a first-order remedy to the problem, and hence have limited correction capabilities. This is because we do not consider the combinations of the cells when we are adding new partitions. In the case, for example, where there are two pairs of close targets whose cells are merged wrongly by Distance Partitioning, the sub-partitioning algorithm presented above would add an additional partition for each of the target pairs (i.e. for each of the wrongly merged cells), but not an additional partition that contains the split versions of both cells. Consideration of all combinations of (the wrongly merged) cells seems to be prohibitive, due to the combinatorial growth in the number of additional partitions. An idea for the cases where there can be more than one wrongly merged cells is to add a single additional partition, which contains split versions of all such cells.

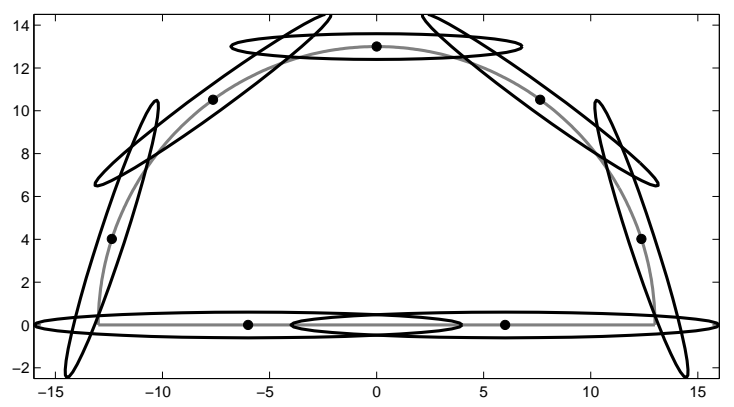

Fig. 2. Birth intensity used in experiments.

\section{TARgET TRACKING SETUP}

The presented tracking approach is exemplified with a laser sensor tracking humans at short distance. In this section the tracking setup is defined for both a pure simulation environment and an experimental realisation with laser data. The targets are modeled as points with state variables

$$
\mathbf{x}_{k}=\left[\begin{array}{llll}
\mathrm{x}_{k} & \mathrm{y}_{k} & \mathrm{v}_{k}^{\mathrm{x}} & \mathrm{v}_{k}^{\mathrm{y}}
\end{array}\right]^{\mathrm{T}},
$$

where $\mathrm{x}_{k}$ and $\mathrm{y}_{k}$ are the planar position coordinates of the target, and $\mathrm{v}_{k}^{\mathrm{x}}$ and $\mathrm{v}_{k}^{\mathrm{y}}$ are the corresponding velocities. The sensor measurements are given in batches of Cartesian $x$ and y coordinates as follows;

$$
\mathbf{z}_{k}^{(j)} \triangleq\left[\begin{array}{ll}
\mathbf{x}_{k}^{(j)} & \mathbf{y}_{k}^{(j)}
\end{array}\right]^{\mathrm{T}}
$$

A constant velocity model [30], with sampling time $T$ is used. In all simulations the probability of detection and probability of survival are set to $p_{\mathrm{D}}=0.99$ and $p_{\mathrm{S}}=0.99$, respectively. The algorithm parameters for the simulation and experiment are given in Table III. The surveillance area is $[-1000,1000](\mathrm{m}) \times[-1000,1000](\mathrm{m})$ for the simulations, and for the real data experiments the surveillance area is a semi circle located at the origin with range $13 \mathrm{~m}$. Unless otherwise stated, in the simulations clutter was generated with a Poisson rate of 10 clutter measurements per scan, and each target generated measurements with a Poisson rate of 10 measurements per scan. The birth intensity in the simulations is

$$
\begin{aligned}
D_{b}(\mathbf{x}) & =0.1 \mathcal{N}\left(\mathbf{x} ; m_{b}, P_{b}\right)+0.1 \mathcal{N}\left(\mathbf{x} ;-m_{b}, P_{b}\right), \\
m_{b} & =[250,250,0,0]^{\mathrm{T}}, P_{b}=\operatorname{diag}([100,100,25,25]) .
\end{aligned}
$$

For the experiments, the birth intensity Gaussian components are illustrated with their corresponding one standard deviation ellipsoids in Fig. 2. Each birth intensity component has a weight $w_{b}^{(j)}=\frac{0.1}{J_{b}}$, where the number of components is $J_{b}=7$. The spawn intensity is

$$
D_{\beta}(\mathbf{x} \mid \mathbf{y})=w_{\beta} \mathcal{N}\left(\mathbf{x} ; \xi, Q_{\beta}\right)
$$

where $\xi$ is the target from which the new target is spawned and the values for $w_{\beta}$ and $Q_{\beta}$ are given in Table III. 
TABLE III

PARAMETER VALUES USED FOR SIMULATIONS (S) AND EXPERIMENTS (E).

\begin{tabular}{|l|l|l|l|l|l|l|}
\hline & $T$ & $Q_{k}$ & $R_{k}$ & $\gamma^{(i)}$ & $w_{\beta}$ & $Q_{\beta}$ \\
\hline $\mathrm{S}$ & 1 & $2^{2} \mathbf{I}_{2}$ & $20^{2} \mathbf{I}_{2}$ & 10 & 0.05 & $\operatorname{blkdiag}_{\left(100 \mathbf{I}_{2}, 400 \mathbf{I}_{2}\right)}$ \\
$\mathrm{E}$ & 0.2 & $2^{2} \mathbf{I}_{2}$ & $0.1^{2} \mathbf{I}_{2}$ & 12 & 0.01 & $0.01 \mathbf{I}_{4}$ \\
\hline
\end{tabular}

\section{Simulation Results}

This section presents results from simulations using the presented extended target tracking method. Section VI-A presents three simulation scenarios that are used several times, and Section VI-B presents a comparison of Distance Partitioning and $K$-means++. In Section VI-C a comparison of Distance Partitioning and Distance Partitioning with Sub-Partition is presented, the results show the increased performance when using Sub-Partition. A comparison between ET-GM-PHD and GM-PHD is presented in Section VI-D, where it is shown that ET-GM-PHD as expected outperforms GM-PHD for extended targets. Section VI-E presents a comparison of ET-GM-PHD and GM-PHD for targets that give rise to at most one measurement per time step. Finally, detailed investigations are carried out about the effects of the possibly unknown parameter $\gamma$ in Section VI-F.

\section{A. True target tracks}

Three different scenarios are used in several simulations. The first two both have two targets. The true $x, y$ positions and the distance between the targets are shown in Fig. 3a and Fig. $3 \mathrm{~b}$. At the closest points the targets are $60 \mathrm{~m}$ and $50 \mathrm{~m}$ apart, respectively. In the third scenario there are four targets in total, the true $\mathrm{x}, \mathrm{y}$ positions are shown in black in Fig. 3c. Around time 50-52 two target tracks cross at a distance of just over $50 \mathrm{~m}$, at time 67 a new target is spawned $20 \mathrm{~m}$ from a previous one. Together the three scenarios present challenges that are typical in multiple target applications. In the simulations, the targets are modeled as points that generate measurements with standard deviation $20 \mathrm{~m}$ in both $\mathrm{x}$ and $\mathrm{y}$ direction. Thus, a measure of target extent can be taken as the two standard deviation measurement covariance ellipses, in this case circles of radius $40 \mathrm{~m}$. In all three scenarios these circles partly overlap when the targets are closest to each other.

\section{B. Comparison of Distance Partitioning and K-means ++}

The scenario in Fig. $3 b$ was used to compare Distance Partitioning to $K$-means ++ . In order to make the comparison as fair as possible, the upper and lower thresholds were set to $K_{\mathrm{L}}=1, K_{\mathrm{U}}=\left|\mathbf{Z}_{k}\right|, \delta_{P_{\mathrm{U}}}=\infty$ and $\delta_{P_{\mathrm{L}}}=0$, respectively. The scenario was simulated with a Poisson rate of 1 and 10 clutter measurements per scan. For each clutter rate, the scenario was simulated 100 times, Fig. 4 shows the resulting sum of weights. At the lower clutter rate, $K$-means++ yields a small positive bias in estimated target number, but the performance is otherwise good. However, at the higher clutter rate the performance using $K$-means ++ is far from acceptable. Distance Partitioning, on the other hand, handles both clutter rates equally well, except for when the targets are close around time 50. Note also that using Distance Partitioning, the sum

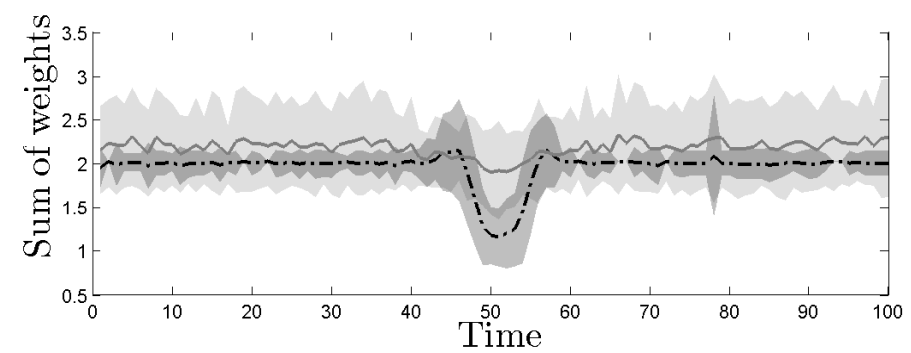

(a) $\beta_{F A, k} V_{s}=1$

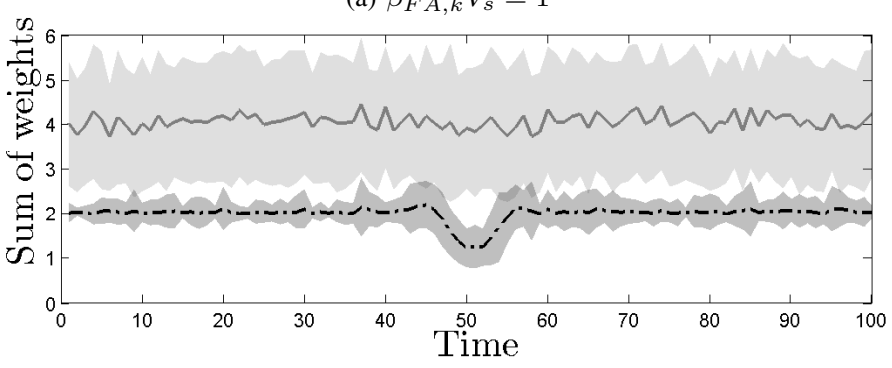

(b) $\beta_{F A, k} V_{s}=10$

Fig. 4. Results from the comparison of Distance Partitioning (black dashdotted line) and $K$-means ++ (gray solid line), the shaded areas correspond to \pm one standard deviation. At the lower clutter rate, $K$-means ++ performs adequately, however at the higher clutter rate the performance is unacceptable. Distance Partitioning on the other hand handles both the lower and higher clutter rate, and has a much smaller uncertainty area.

of weights uncertainty area is considerably smaller. The case of close targets is investigated further in the next subsection, using the countermeasure introduced in Section IV-C.

\section{Benefits of Sub-Partition}

As was noted in Section IV-C, as well as in previous work [20], using only Distance Partitioning to obtain a subset of all possible partitions is insufficient when the extended targets are spatially close. For this reason, Sub-Partition was introduced to obtain more partitions. In this section, we present results from simulations that compare the performance of ET-GMPHD tracking with partitions computed using only Distance Partitioning and with partitions computed using Distance Partitioning and Sub-Partition. The scenarios in Fig. 3a and Fig. 3b are considered.

Each scenario was simulated 100 times with a constant expected number of measurements per target $(\gamma(\cdot)=\gamma)$ of 5, 10 and 20, respectively. Fig. 5 shows the resulting sum of weights of the ET-GM-PHD algorithm. As can be seen, using Sub-Partition the average sum of weights is closer to the true number of targets. This is especially clear for targets that generate more measurements per time step, i.e. when $\gamma$ is higher.

\section{Comparison with GM-PHD}

This section presents results from a simulation comparison of ET-GM-PHD and GM-PHD. Note here that the GM-PHD filter is applied naively to the simulated measurements, i.e. it is applied under the (false) assumption that each target produces at most one measurement per time step. The scenario in Fig. 3c is considered. 

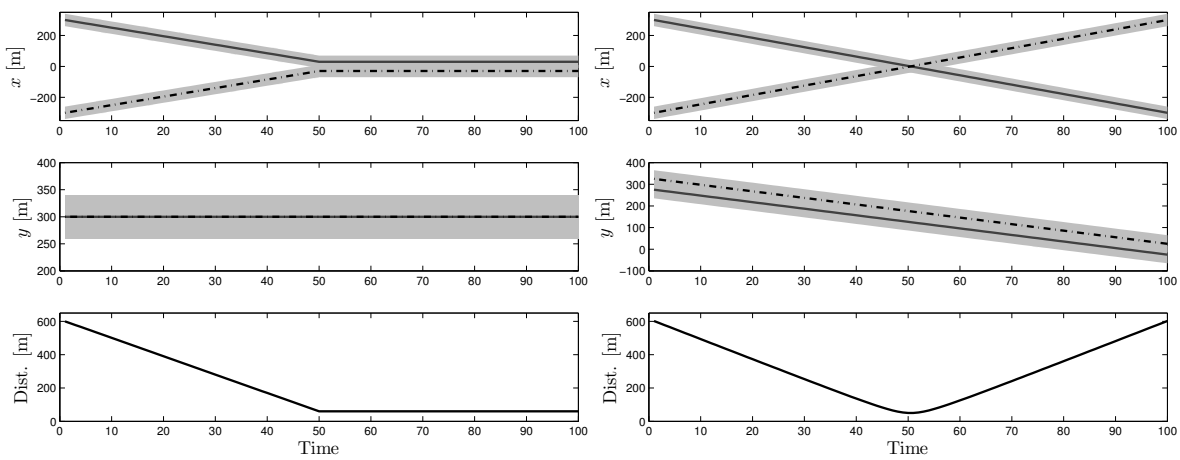

(a)

(b)
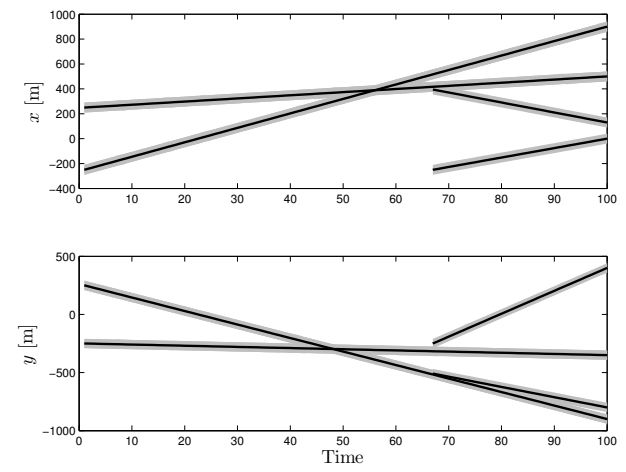

(c)

Fig. 3. (a) Two targets move closer to each other and then stand still at a distance of $60 \mathrm{~m}$ apart. Note that the true $\mathrm{y}$ position was $300 \mathrm{~m}$ for both targets for the entire simulation. (b) Two targets cross paths, at the closest point they are $50 \mathrm{~m}$ apart. (c) Four targets, with a target spawning event at time 67 . The $x$ and $y$ positions are shown as lines, the light gray shaded areas show the target extent, taken as two measurement noise standard deviations (40m). In (a) and (b), the bottom row shows the distance between the two targets over time.

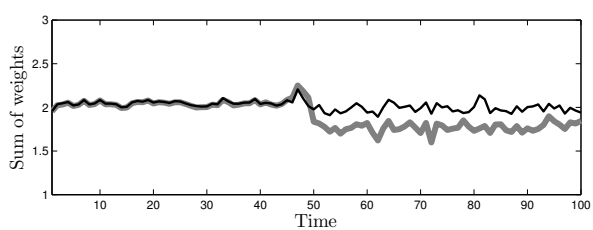

(a) $\gamma=5$

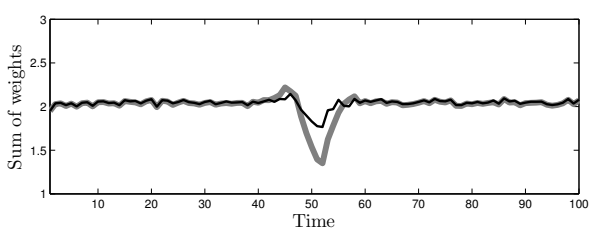

(d) $\gamma=5$

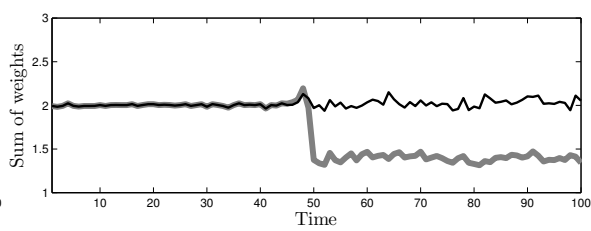

(b) $\gamma=10$

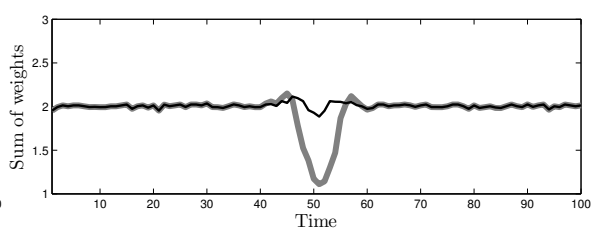

(e) $\gamma=10$

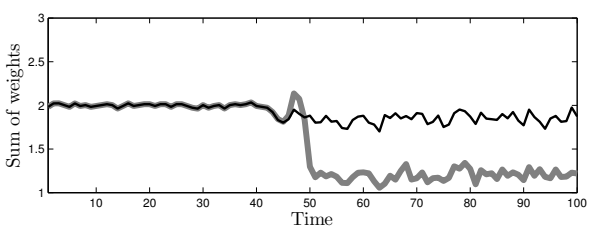

(c) $\gamma=20$

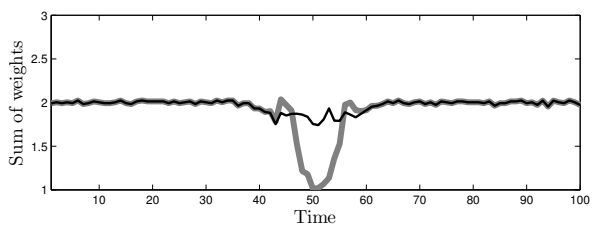

(f) $\gamma=20$

Fig. 5. Simulation results for two of the scenarios in Fig. 3, comparing different partitioning methods for different values of the expected number of measurements $\gamma$. The top row, (a), (b) and (c), is for the true tracks in Fig. 3a. The bottom row, (d), (e) and (f), is for the true tracks in Fig. 3b. Black shows Distance Partitioning with Sub-Partition, gray is only Distance Partitioning. It is clear from the plots that using Sub-Partition gives significantly better results, especially when $\gamma$ is higher.

In total 100 Monte Carlo simulations were performed, each with new measurement noise and clutter measurements. The results are shown in Fig. 6a and Fig. 6b, which show the corresponding multi-target measure optimal subpattern assignment metric (OSPA) [31], and the cardinality, respectively. In the OSPA metric the parameters are set to $p=2$, corresponding to using the 2-norm which is a standard choice, and $c=60$, corresponding to a maximum error equal to three measurement noise standard deviations. Here, the cardinality is computed as $\sum_{j=1}^{J_{k \mid k}} w_{k \mid k}^{(j)}$. This sum can be rounded to obtain an integer estimate of target number [18].

It is evident from the two figures that the presented ET-GMPHD significantly outperforms the standard GM-PHD, which does not take into account the possibility of the multiple measurements from single targets. The main difference between the two filters is the estimation of cardinality, i.e. the number of targets. The ET-GM-PHD-filter correctly estimates the cardinality with the exception of when the new target is spawned - after time 67 there is a small dip in the mean estimated cardinality, even though Sub-Partition is used. The reason for this is that the targets are only $20 \mathrm{~m}$ apart. With the target extension being a circle of $40 \mathrm{~m}$ radius, at $20 \mathrm{~m}$ distance the measurements overlap significantly and the probability that the new target's measurements were also generated by the old target, as computed in (12e), is large. As the targets separate, this probability decreases and the ET-GM-PHD filter recovers the correct cardinality. It should still be noted that, in reality, where the targets would probably be rigid bodies, this type of very close situation is highly unlikely and the results of the ET-GM-PHD filter with Sub-Partition would be close to those presented in Section VI-C.

\section{E. Standard single measurement targets}

This section investigates how ET-GM-PHD handles standard targets that produce at most one measurement per time step, in comparison to standard GM-PHD which is designed under the standard target measurement generation assumption. Note that the measurement set cardinality distribution (i.e. the probability mass function for the number of measurements generated by a target) for a standard target contains only a single nonzero 


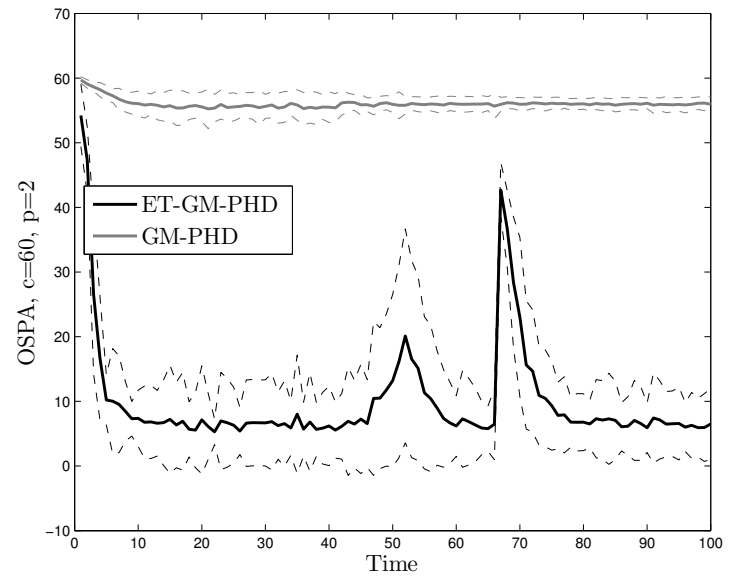

(a)

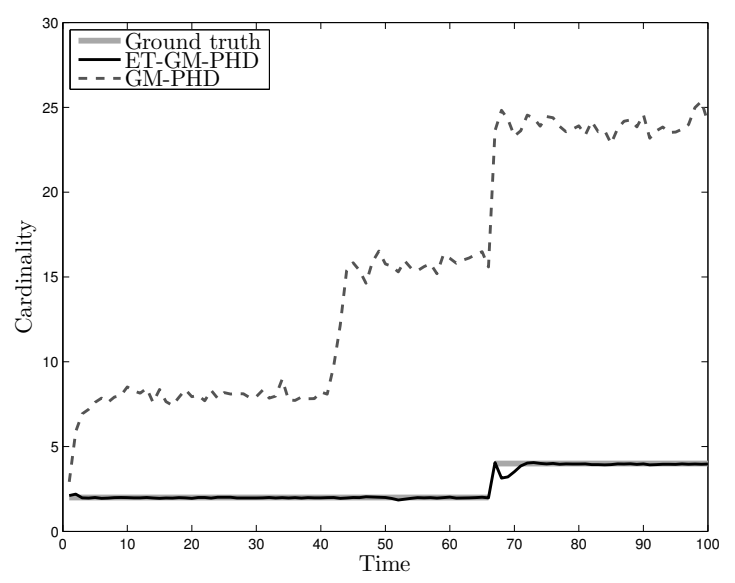

(b)

Fig. 6. Results from multiple target tracking using the true tracks in Fig. 3c. (a) Mean OSPA (solid lines) \pm 1 standard deviation (dashed lines). (b) Mean cardinality compared to the true cardinality.

element ${ }^{2}$ at cardinality 1 , which is impossible to model with a Poisson distribution underlying the ET-GM-PHD filter. Hence, the case where each target generates measurements whose number is Poisson distributed with rate $\gamma=1$ is very different from the standard target measurement generation.

Four targets were simulated in 100 Monte Carlo simulations, and all the targets were separated, i.e. there were no track crossings or new target spawn. Initially, in the ET-GM-PHD filter, $\gamma^{(j)}$ are all set as $\gamma^{(j)}=1$ in the comparison. The average sum of weights and the average number of extracted targets (obtained by rounding the weights to the nearest integer) for the algorithms are shown in Fig. 7a and Fig. 7b respectively. As is shown, the sum of weights and number of extracted targets are too large for the ET-GM-PHD filter. The reason for this is that when the expected number of measurements per target (i.e. $\gamma^{(j)}$ ) is small, the effective probability of detection ${ }^{3}$

$$
p_{\mathrm{D}, \mathrm{eff}}^{(j)}=\left(1-e^{-\gamma^{(j)}}\right) p_{\mathrm{D}}^{(j)}
$$

becomes significantly smaller than one. For example, the case $\gamma^{(j)}=1$ and $p_{\mathrm{D}}^{(j)}=0.99$ gives $p_{\mathrm{D} \text {,eff }}^{(j)}=0.6258$. This low effective probability of detection is what causes the weights in the ET-GM-PHD filter to become too large.

Actually, this problem has been seen to be inherited by the ET-GM-PHD filter from the standard PHD filter. We here give a simple explanation to the problem with low (effective) probability of detection in the PHD filter. Assuming no false alarms, and a single target with existence probability $p_{\mathrm{E}}$, ideally a single detection should cause the expected number of targets to be unity. However, applying the standard PHD formulae to this simple example, one can calculate the updated expected number of targets to be $1+p_{\mathrm{E}}\left(1-p_{\mathrm{D}}\right)$ whose positive bias increases as $p_{\mathrm{D}}$ decreases. We have seen that when the (effective) probability of detection is low, the increase in

\footnotetext{
${ }^{2}$ Note that a standard target always generates a single measurement Whether no measurements or a single measurement is obtained from the standard target is determined by the detection process.

${ }^{3}$ More correctly, $p_{\mathrm{D}, \text { eff }}^{(j)}$ in $(29)$ is the probability of the event that at least one measurement from the $(j$ th) target is obtained by the sensor.
}

$\sum_{j=1}^{J_{k \mid k}} w_{k \mid k}^{(j)}$ is a manifestation of this type of sensitivity of the PHD type filters. ${ }^{4}$ A similar sensitivity issue is mentioned in [33] for the case of no detection.

This problem can be overcome by increasing $\gamma^{(j)}$ slightly in the ET-GM-PHD filter, e.g. $\gamma^{(j)}=2$ gives $p_{\mathrm{D} \text {,eff }}^{j}=0.8560$ which gives sum of weights and number of extracted targets that better match the results from GM-PHD, see Fig. 7c and Fig. 7d. Using $\gamma^{(j)}=3$ gives results that are more or less identical to GM-PHD, thus a conclusion that can be drawn is that when tracking standard targets with an ET-GM-PHD filter, the parameter $\gamma^{(j)}$ should not be set too small. The following subsection investigates the issue of selecting the parameter $\gamma$ in more detail.

\section{F. Unknown expected number of measurements $\gamma$}

In the simulations above, the parameters $\gamma=\gamma^{(j)}$ were assumed to be known a priori. Further, in Section IV-C where Sub-Partition is presented, the knowledge of the Poisson rate $\gamma$ was used to determine whether a cell should be split or not to create an additional partition. In this section, some scenarios where $\gamma$ is not known a priori are investigated. For the sake of clarity, $\gamma$ is used to denote the true Poisson rate with which measurements were generated, and $\hat{\gamma}$ is used to denote the corresponding parameter in the ET-GM-PHD-filter.

In many real world scenarios, the number of measurements generated by a target is dependent on the distance between the target and the sensor. Typically, the longer the distance, the lower the number of measurements generated by the targets. This is true for sensors such as laser range sensors, radars and even cameras. Thus, it is of interest to evaluate the ETGM-PHD-filter in a scenario where the number of generated measurements varies with the target to sensor distance. This is simulated in Section VI-F1, where the ET-GM-PHD filter is compared for the cases when the parameter $\hat{\gamma}$ is constant, and when the parameter is modeled as distance varying. Section VI-F2 presents results from simulations where the

\footnotetext{
${ }^{4}$ Some extreme versions of this phenomenon for lower $P_{\mathrm{D}}$ values are illustrated and investigated in detail in the recent work [32].
} 


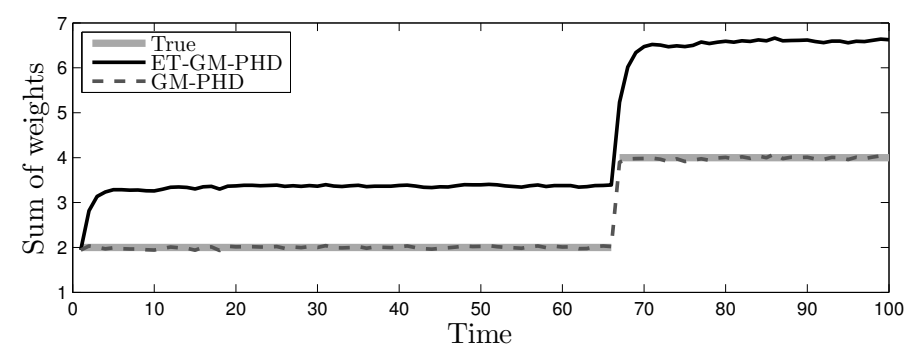

(a) $\gamma^{(j)}=1$

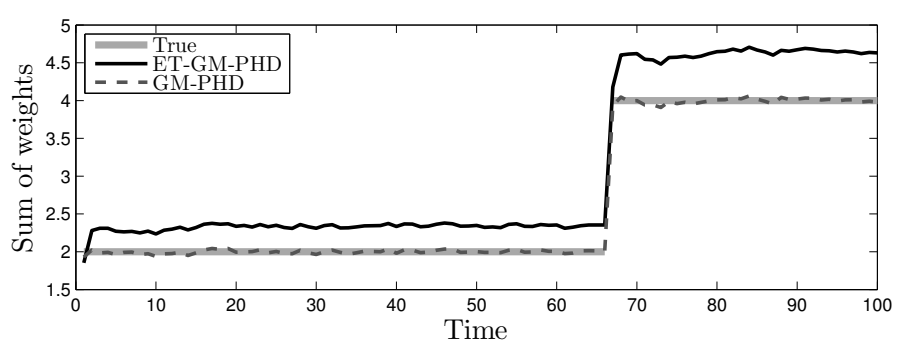

(c) $\gamma^{(j)}=2$

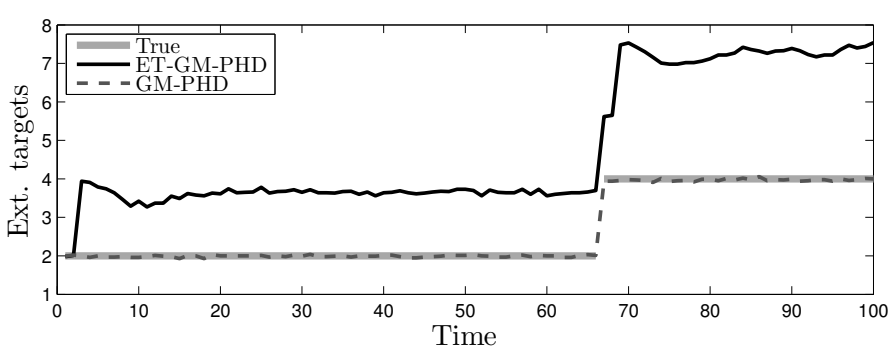

(b) $\gamma^{(j)}=1$

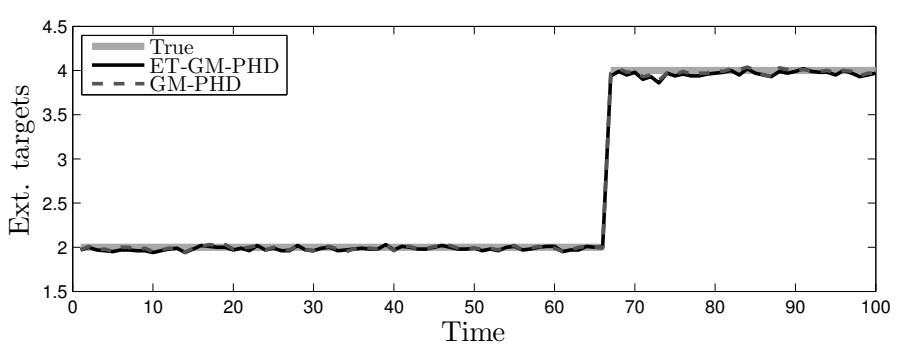

(d) $\gamma^{(j)}=2$

Fig. 7. Simulation results, comparison of ET-GM-PHD and GM-PHD for standard targets that produce at most one measurement per time step. The top row shows results when the parameter $\gamma^{(j)}$ is set to one, the bottom row shows results when it is set to two. Due to the low effective probability of detection, the ET-GM-PHD weights become too large, resulting in sum of weights larger than the true number of targets. When each weight is rounded to the nearest integer to extract targets, results for $\gamma^{(j)}=2$ gives the correct number of extracted targets.

parameter $\hat{\gamma}$ is set incorrectly, and Section VI-F3 presents results with targets of different sizes. Finally, Section VI-F4 presents a discussion about the results from the simulations, and supplies some guidelines into the selection of $\hat{\gamma}$.

1) Distance varying $\gamma$ : A scenario was constructed where a target moved such that the target to sensor distance first decreased, and then increased. The sensor was simulated such that the true parameter $\gamma$ depended on the target to sensor distance $\rho$ as follows.

$$
\gamma(\rho)= \begin{cases}1, & \text { if } \rho>400 \mathrm{~m} \\ \lfloor-0.08 \rho+33.5\rfloor, & \text { if } 100 \mathrm{~m} \leq \rho \leq 400 \mathrm{~m} \\ 25, & \rho<100 \mathrm{~m}\end{cases}
$$

where $\lfloor\cdot\rfloor$ is the floor function. Thus, at distances larger than $400 \mathrm{~m}$, with $p_{\mathrm{D}}^{(j)}=0.99$, the effective probability of detection is only 0.6258 (as in the previous subsection). Note that the scenario is different from a target that always generates one measurement, which is detected with probability $p_{\mathrm{D}}^{(j)}=0.99$.

Monte Carlo simulations were made with two ET-GM-PHDfilters: one with constant value $\hat{\gamma}=10$ and another where $\hat{\gamma}$ was selected to be dependent on the state of the targets via the function (30). The results are shown in Fig. 8. For constant $\hat{\gamma}$, the number of targets is underestimated when the true $\gamma$ is low. This is due to the fact that the filter expects a target to generate more measurements, and thus the likelihood that some small number of measurements are all clutter is higher. However, at distances $\rho$ such that $\gamma(\rho)>5, \hat{\gamma}=10$ works quite well. When the model (30) for distance dependent $\gamma$ is assumed known, the results are much more reasonable and acceptable. The only, and possibly negligible, drawback seems to be the number of targets being slightly overestimated. There are two main reasons for this. The first reason is the low effective probability of detection when $\hat{\gamma}$ is low. When $\hat{\gamma}$ becomes smaller than 5 , this behavior is more evident. The second reason is that the clutter falling into the region $\rho>400 \mathrm{~m}$ (i.e. when the true parameter is $\gamma=1$ ) is interpreted as targets to some extent, which causes a positive, though small, bias in the estimation of number of targets. In that region, the target behavior is fairly similar to the clutter behavior which results in some Gaussian components with small weights surviving until the situation is resolved.

2) Incorrect $\gamma$ parameter: In this simulation study, the target tracks in Fig. 3b were used. Each target generated measurements with a Poisson rate of $\gamma=20$ and eleven different ET-GM-PHD-filters, each using a different $\hat{\gamma}$ value, were run. The set of $\hat{\gamma}$ values used is given as

$$
\hat{\gamma}=10,12, \ldots, 28,30
$$

The results, in terms of the sum of weights averaged over the Monte Carlo runs, are shown in Fig. 9. The figure shows that for sufficiently separated targets, the ET-GM-PHD-filter correctly estimates the number of targets for all values of $\hat{\gamma}$. However, for spatially close targets, the ET-GM-PHD-filter overestimates the number of targets when $\hat{\gamma}$ is set too low, and underestimates the number of targets when $\hat{\gamma}$ is set too high. This result is actually expected, and is a direct consequence of the simple Sub-Partition algorithm which is used. When $\hat{\gamma}$ is too low, Sub-Partition creates an additional partition with too many cells, causing the overestimation of number of targets. Conversely, when $\hat{\gamma}$ is too high, Sub-Partition does not create partitions with sufficient number of cells, causing the underestimation of number of targets. It is very important to note here that Sub-Partition runs even when the targets are well separated and does not cause any overestimation. Our 


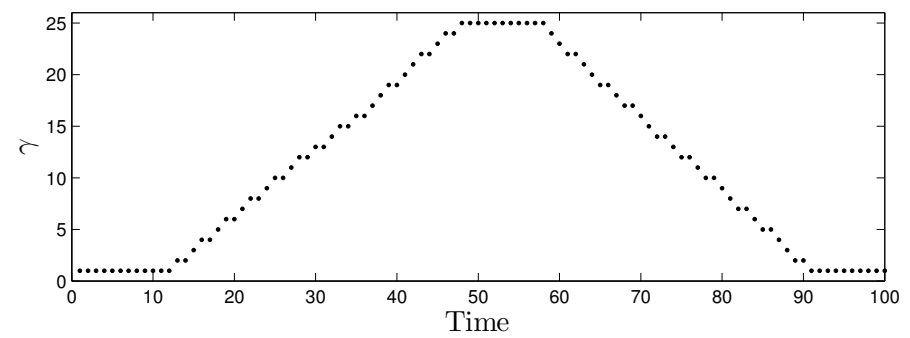

(a)

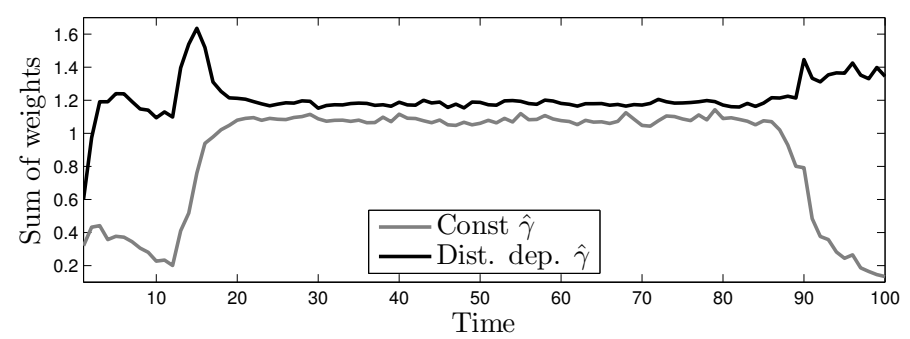

(b)

Fig. 8. Results from the simulation scenario where $\gamma$ is dependent on the target to sensor distance. In (a), the true $\gamma$ is plotted against time, and in (b) the mean sum of weights is plotted against time. The ET-GM-PHD-filter is compared for the cases when the parameter $\hat{\gamma}$ is held constant (gray) or is set to the true distance dependent value (black). The correct target number is one, thus the sum of weights should be around one. In both cases, at the beginning and the end of the scenario when the distance is largest and $\gamma=1$, tracking performance gets worse.

observations show that this is a result of the fact that additional partitions created (when $\hat{\gamma}$ is selected too low) cannot win over single target partitions when the targets measurements are distributed in a blob shape. It is only when the two targets approach each other, resulting in an eight-shaped cluster of measurements, that the additional partition can gain dominance over the single target partition. This property, though not proved mathematically, is considered to be a manifestation of the Poisson property and the Gaussianity assumptions underlying the measurements.

If the cardinality estimates of the algorithms are rounded to the nearest integer, an interesting property observed with Fig. 9 is that no cardinality error appears for the cases that satisfy

$$
\hat{\gamma}-\sqrt{\hat{\gamma}} \leq \gamma \leq \hat{\gamma}+\sqrt{\hat{\gamma}}
$$

Thus, when the true parameter $\gamma$ lies in the interval determined by the mean $(\hat{\gamma}) \pm$ one standard deviation $(\sqrt{\hat{\gamma}})$, cardinality is expected to be estimated correctly even for close targets.

3) Targets of different size: In many scenarios, it is possible to encounter multiple targets of different sizes, thus producing a different number of measurements. This means that two targets would not have the same Poisson rate $\gamma$. In this section, results are presented for a scenario with two targets with measurement generating Poisson rates of 10 and 20, respectively. In Monte Carlo simulations, three ET-GM-PHDfilters were run with the parameter $\hat{\gamma}$ set to 10,15 and 20 , respectively. This corresponds to using either the true value of the smaller target, the mean of both, or the true value of the larger target. The results, in terms of average sum of weights over time are shown in Fig. 10. When the targets

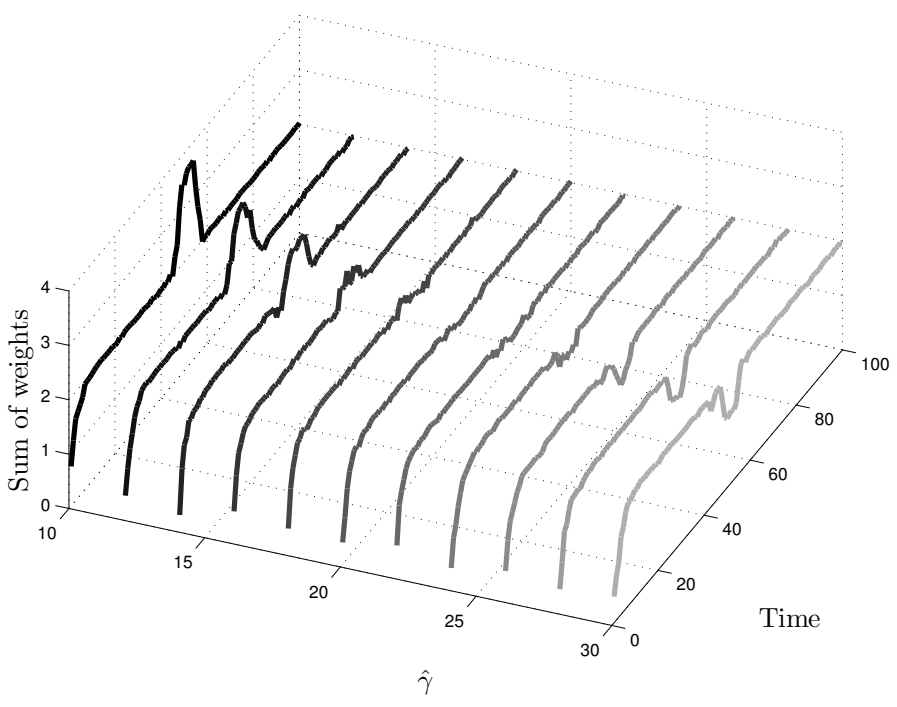

Fig. 9. Simulation results for various values of the ET-GM-PHD-filter parameter $\hat{\gamma}$. There are two targets, the true Poisson rate used to generate measurements for both targets was $\gamma=20$.

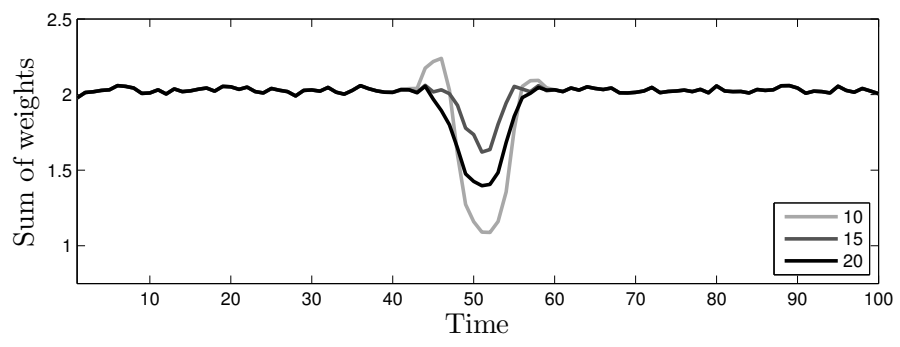

Fig. 10. Simulation results from a scenario with two targets of different sizes. The two targets have the true Poisson rates $\gamma=10$ and $\gamma=20$, respectively. The legend refers to the filter parameter $\hat{\gamma}$.

are spatially separated, all three filters perform equally well. However, when the targets are spatially close, the target with $\hat{\gamma}$ set to the mean of the true $\gamma \mathrm{s}$ performs better than the others.

4) Discussion: The simulation results above show that the ET-GM-PHD-filter works well even when $\hat{\gamma} \neq \gamma$, except when $\gamma<5$ or targets are spatially close. For $\gamma<5$, the filter is more sensitive, and a correct value for $\hat{\gamma}$ is increasingly important. For targets that are spatially close, it is important for $\hat{\gamma}$ to be a good estimate of $\gamma$, since the Sub-Partition algorithm relies on $\hat{\gamma}$. When such a good estimate is unavailable, a more advanced sub-partitioning algorithm seems to be necessary for robustness. With the proposed Sub-Partition procedure, our findings support the intuitive conclusion that the true parameter $\gamma$ should be in one standard deviation uncertainty region around the mean $\hat{\gamma}$ of the Poisson distribution for a reasonable performance for close targets.

The simulation with different target sizes shows that the close target case in this example is harder to tackle than the others. A possible solution is to adaptively estimate the parameters $\hat{\gamma}$ for each Gaussian mixture component, based on the previous measurements. Another solution, which is possibly more straightforward, is to use a state dependent $\hat{\gamma}$ parameter, where the state contains information about the target extent, which can readily be estimated, see e.g. [5], [6], 
[13]-[15]. Using the estimated shape and size, and a model of the sensor that is used, $\hat{\gamma}$ can then be estimated with reasonable accuracy. This has indeed recently been performed using an ET-GM-PHD-filter [13].

\section{EXPERIMENT RESULTS}

This section presents results from experiments with data from two data sets acquired with a laser range sensor. The experiments are included more as a proof of concept and as a potential application, rather than as an exhaustive evaluation of the presented target tracking filter. The measurements were collected using a SICK LMS laser range sensor. The sensor measures range every $0.5^{\circ}$ over a $180^{\circ}$ surveillance area. Ranges shorter than $13 \mathrm{~m}$ were converted to $(\mathrm{x}, \mathrm{y})$ measurements using a polar to Cartesian transformation.

The two data sets contain 411 and 400 laser range sweeps in total, respectively. During the data collection humans moved through the surveillance area, entering the surveillance area at different times. The laser sensor was at the waist level of the humans.

Because there is no ground truth available it is difficult to obtain a definite measure of target tracking quality, however by examining the raw data we were able to observe the true cardinality, which can thus be compared to the estimated cardinality.

Section VII-A presents results from an experiment with spatially close targets, and Section VII-B presents results from an experiment with both spatially close targets and occlusion.

\section{A. Experiment with close targets}

In this experiment, a data set containing 411 laser range scans was used. The data set contains two human targets that repeatedly move towards and away from each other, moving right next to each other at several times. The two targets passed each other at close distance moving in the opposite direction, representing instances in time when the targets were close for short periods of time. The targets also moved close to each other moving in the same direction, representing instances in time when the targets were close for longer periods of time.

The locations of the extracted Gaussian components are shown in Fig. 11a, the number of extracted targets is shown in Fig. $11 \mathrm{~b}$ and the sum of weights are shown in Fig. 11c. Around time 320 there is a decrease in the number of extracted targets for three time steps, in all other situations the filter handles the two targets without problem. Thus, using Sub-Partition with $K$-means as $\operatorname{split}(\cdot, \cdot)$ function, the ET-GM-PHD filter can be said to handle most of the spatially close target cases.

\section{B. Experiment with occlusion}

In this experiment, a dataset containing 400 laser range scans was used. The data set contains four human targets that move through the surveillance area, however there are at most three targets present at any one time. The first target enters the surveillance area at time $k=22$ and proceeds to the center of the surveillance area where he remains still for the remainder of the experiment. The second target enters the surveillance

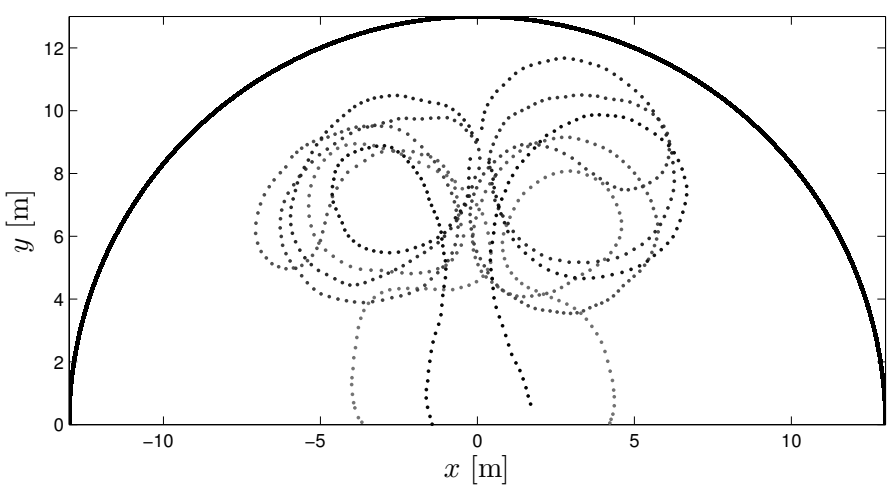

(a)

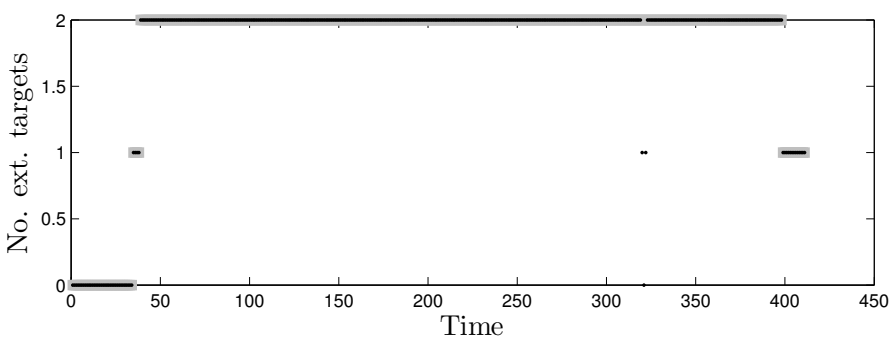

(b)

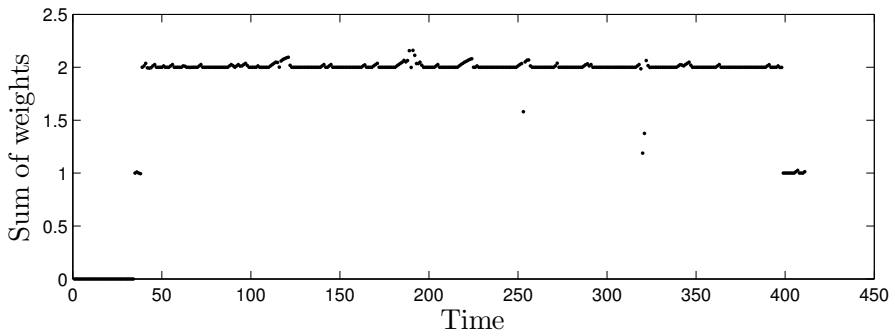

(c)

Fig. 11. Experiment results, two human targets moving close to each other. Note that in (a) the gray scale indicates the time line, lighter gray is earlier time steps, darker is later time steps. In (b), the number of extracted targets (black) is compared to the ground truth (gray). In (c) the sum of weights is shown. Around time 320 the cardinality is underestimated for three time steps.

area at time $k=38$ and repeatedly moves in front of and behind the first target. The third target enters and exits at time $k=283$ and $k=310$, respectively. The last target enters and exits at time $k=345$ and $k=362$, respectively.

This case requires a state dependent (i.e. variable) probability of detection $p_{\mathrm{D}}(\cdot)$ selection for the targets. Otherwise, i.e. with a constant probability of detection assumption, when a target is occluded, this would be interpreted as the exit of the target from the area of surveillance while it is only the disappearance of the target behind another. The variable $p_{\mathrm{D}}$ is modeled as a function of the mean, covariance and the weights of the Gaussian components. The intuition behind this idea is that the knowledge of the targets that are present, i.e. the knowledge of the estimated Gaussian components of the PHD, can be used to determine what parts of the surveillance area are likely to be in view of the sensor, and which parts are not. Leaving the details of the variable $p_{\mathrm{D}}$ calculation to Appendix B, we present the results below.

The locations of the extracted Gaussian components are shown in Fig. 12a, the number of extracted targets is shown in 


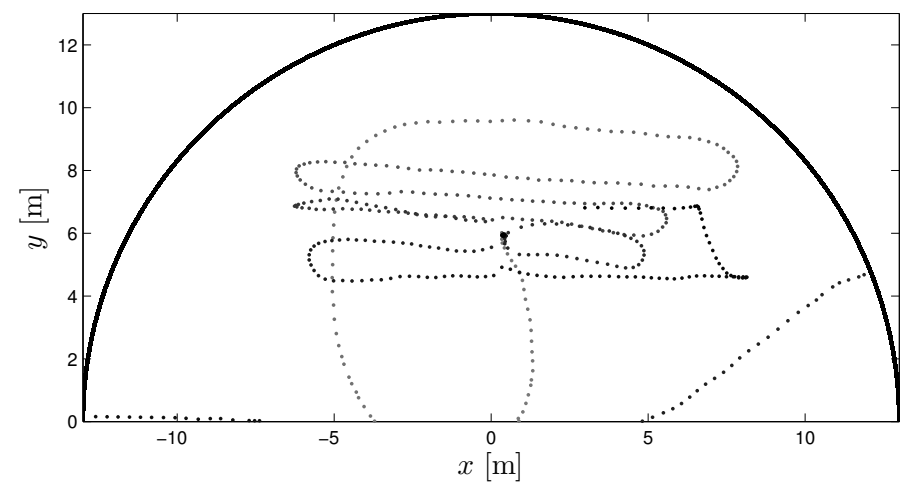

(a)

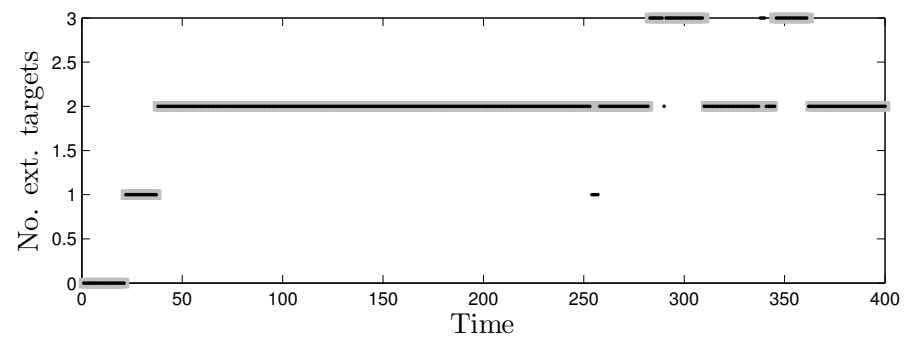

(b)

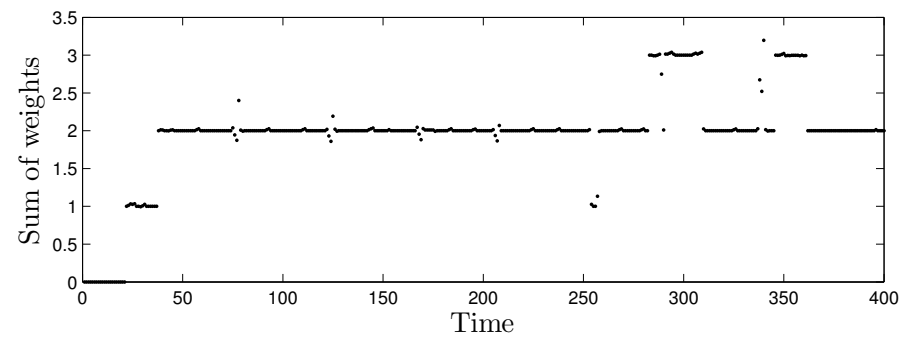

(c)

Fig. 12. Experiment results, targets moving in and out of occluded regions of the surveillance area. Note that in (a) the gray scale indicates the time line, lighter gray is earlier time steps, darker is later time steps. In (b), the number of extracted targets (black) is compared to the ground truth (gray). In (c) the sum of weights is shown.

Fig. 12b and the sum of weights are shown in Fig. 12c. In total, there are six situations where one target is occluded by another. The extracted number of targets is incorrect in two of these situations, where the occluded target is spatially very close to (right next to) the target which is causing the occlusion. The ET-GM-PHD filter correctly estimates the cardinality in four out of six occlusions.

Thus, using the suggested variable $p_{\mathrm{D}}$, the filter can correctly predict the target while it is occluded, provided that it is not very close to another target while the occlusion is happening. If $\sum_{j=1}^{J_{k \mid k}} w_{k \mid k}^{(j)}$ is rounded to the nearest integer there is no cardinality error for the first four occlusions. However, as the target exits the occluded area there is a "jump" in $\sum_{j=1}^{J_{k \mid k}} w_{k \mid k}^{(j)}$ around times $k=75, k=125$, $k=175$ and $k=210$, see Fig. 12c. We have seen that this "jumping" behavior is caused by the sensitivity of the cardinality estimates of the PHD filter to detections when $p_{\mathrm{D}}^{(j)}$ is set to a low value, which is the case when the target is half occluded while it gets out of the occluded region. This is the same phenomenon observed with low effective probability of detection in Section VI-E.

\section{CONCLUSIONS}

In this paper a Gaussian mixture implementation of the probability hypothesis density filter for tracking extended targets was presented. It was shown that all possible partitions of the measurement set does not need to be considered, instead it is sufficient to consider a subset of partitions, as long as this subset contains the most probable partitions. A simple method for finding this subset of all measurement set partitions was described. This partitioning method is complemented with a sub-partitioning strategy to handle the cases that involve close targets better. Simulations and experiments have shown that the proposed filter is capable of tracking extended targets in cluttered measurements. The number of targets is estimated correctly even for most of the cases when tracks are close. The detailed investigations carried out gave some guidelines about the selection of the Poisson rate parameter for the cases when it is unknown. Using inhomogeneous detection probabilities in the surveillance region, it was shown that targets can be tracked as they move through occluded parts of the surveillance area.

\section{Future WORK}

In recent work, a cardinalized PHD filter [34] for extended targets has been presented [32]. This filter has less sensitive estimates of the number of targets. Initial steps have also been taken towards including estimation of target extent in the ETGM-PHD-filter [13]. More work is needed in both of these research directions.

A further interesting research can be to see the potential use of the partitioning algorithms presented in this work with more conventional multiple target tracking algorithms. A comparison of such algorithms with the ET-GM-PHD filter can illustrate the advantage coming from the use of the random set framework.

\section{APPENDIX A \\ PROOF OF THEOREM 1}

The proof is composed of two parts.

- We first prove that there is a partition satisfying the conditions of the theorem. The proof is constructive. Consider the algorithm in Table IV. In the algorithm, one first forms a partition formed of singleton sets of the individual measurements and then combine the cells of this cluster until conditions of the theorem are satisfied.

- We need to prove that the partition satisfying the conditions of the theorem is unique. The proof is by contradiction. Suppose that there are two different partitions $\mathrm{p}_{i}$ and $\mathrm{p}_{j}$ satisfying the conditions of the theorem. Then, there must exist (at least) one measurement $\mathbf{z}^{(m)} \in \mathbf{Z}$ such that the cells $W_{m_{i}}^{i} \ni \mathbf{z}^{(m)}$ and $W_{m_{j}}^{j} \ni \mathbf{z}^{(m)}$ are different, i.e., $W_{m_{i}}^{i} \neq W_{m_{j}}^{j}$. This requires (at least) a single measurement $\mathbf{z}^{(n)} \in \mathbf{Z}$ that is in one of $W_{m_{i}}^{i}, W_{m_{j}}^{j}$ but not in the other. Without loss of generality, suppose 
TABLE IV

FIND PARTITION p THAT SATISFIES THE CONDITIONS OF THEOREM

Require: Set of measurements $\mathbf{Z}=\left\{\mathbf{z}^{(1)}, \mathbf{z}^{(2)}, \ldots, \mathbf{z}^{\left(N_{z}\right)}\right\}$, where $N_{z}$ is the number of measurements.

1: Set $\mathrm{p}^{0}=\left\{\left\{\mathbf{z}^{(1)}\right\},\left\{\mathbf{z}^{(2)}\right\}, \ldots,\left\{\mathbf{z}^{\left(N_{z}\right)}\right\}\right\}$ i.e., set $W_{j}^{0}=\left\{\mathbf{z}^{(j)}\right\}$ for $j=1, \ldots, N_{z}$.

2: Set $i=1$.

3: Calculate all the pairwise distances between the cells of $\mathrm{p}_{i-1}$ as

$$
\eta_{s t}^{i-1}=\min _{\substack{\mathbf{z}^{(m)} \in W_{s}^{i-1} \\ \mathbf{z}^{(n)} \in W_{t}^{i-1}}} \mathbf{d}\left(\mathbf{z}^{(m)}, \mathbf{z}^{(n)}\right)
$$

4: If $\min _{1<s \neq t<\left|\mathrm{p}^{i-1}\right|} \eta_{s t}^{i-1}>d_{\ell}$, then stop the algorithm, since $\mathrm{p}^{i-1}$ is a partition satisfying the conditions of the theorem.

5: Otherwise, combine all cells that satisfy $\eta_{s t}^{i-1} \leq d_{\ell}$ to form a single cell.

6: Set $\mathrm{p}^{i}$ to be the set of cells obtained in Step 5 .

7: Set $i=i+1$ and go to Step 3 .

$\mathbf{z}^{(n)} \in W_{m_{i}}^{i}$ and $\mathbf{z}^{(n)} \notin W_{m_{j}}^{j}$. Since both $\mathbf{z}^{(m)}$ and $\mathbf{z}^{(n)}$ are in $W_{m_{i}}^{i}$, there must exist a (possibly empty) subset $\left\{\mathbf{z}^{\left(r_{1}\right)}, \mathbf{z}^{\left(r_{2}\right)}, \ldots, \mathbf{z}^{\left(r_{R}\right)}\right\} \subset W_{m_{i}}^{i} \backslash\left\{\mathbf{z}^{(m)}, \mathbf{z}^{(n)}\right\}$ such that the following conditions hold.

$$
\begin{aligned}
\mathbf{d}\left(\mathbf{z}^{(m)}, \mathbf{z}^{\left(r_{1}\right)}\right) & \leq d_{\ell} \\
\mathbf{d}\left(\mathbf{z}^{\left(r_{s}\right)}, \mathbf{z}^{\left(r_{s+1}\right)}\right) & \leq d_{\ell} \quad s=1,2, \ldots, R-1 \\
\mathbf{d}\left(\mathbf{z}^{\left(r_{R}\right)}, \mathbf{z}^{(n)}\right) & \leq d_{\ell}
\end{aligned}
$$

However, (34) implies that the measurements $\left\{\mathbf{z}^{(m)}, \mathbf{z}^{\left(r_{1}\right)}, \mathbf{z}^{\left(r_{2}\right)}, \ldots, \mathbf{z}^{\left(r_{R}\right)}, \mathbf{z}^{(n)}\right\}$ should all be in the same cell. For $\mathrm{p}_{j}$, this is the cell $W_{m_{j}}^{j} \ni \mathbf{z}^{(m)}$, which contradicts the fact that $\mathbf{z}^{(n)} \notin W_{m_{j}}^{j}$. Thus, the initial assumption that there are two different partitions satisfying the conditions of the theorem must be wrong. The proof is complete.

\section{APPENDIX B \\ VARIAble Probability of Detection FOR THE LASER SENSOR}

The variable probability of detection function reduces $p_{\mathrm{D}}$ behind (i.e. at larger range from the sensor) each component of the PHD.

For a given point $\mathbf{x}$ in the surveillance area, the probability of detection $p_{\mathrm{D}}(\mathbf{x})$ is computed as

$$
\begin{aligned}
p_{\mathrm{D}}(\mathbf{x}) & =\max \left(p_{\mathrm{D}, \min }, p_{\mathrm{D}}^{\mathrm{v}}\right) \\
p_{\mathrm{D}}^{\mathrm{v}} & =p_{\mathrm{D}, 0}-\sum_{i: r>r^{(i)}} w^{(i)} \sqrt{\frac{\sigma_{s}}{\bar{\sigma}_{\varphi^{(i)}}}} \exp \left(\frac{-\left(\varphi-\varphi^{(i)}\right)^{2}}{2 \bar{\sigma}_{\varphi^{(i)}}}\right)
\end{aligned}
$$

where

- $p_{\mathrm{D}, \min }$ is the minimum probability of detection value a target can have;

- $p_{\mathrm{D}, 0}$ is the nominal probability of detection of the targets when they are not occluded;

- $r$ and $\varphi$ are the range and bearing, respectively, from the sensor to the point $\mathbf{x}$;

- $r^{(i)}$ and $\varphi^{(i)}$ are the range and bearing, respectively, from the sensor to the $i$ th Gaussian component;

- $w^{(i)}$ is the weight of the $i$ th component;
- $\bar{\sigma}_{\varphi^{(i)}}$ is defined as

$$
\bar{\sigma}_{\varphi^{(i)}} \triangleq \begin{cases}\sigma_{\max }, & \text { if } \sigma_{\varphi^{(i)}}>\sigma_{\max } \\ \sigma_{\min }, & \text { if } \sigma_{\varphi^{(i)}}<\sigma_{\min } \\ \sigma_{\varphi^{(i)}}, & \text { otherwise }\end{cases}
$$

where $\sigma_{\varphi^{(i)}}$ is the bearing standard deviation of the $i$ th component given as

$$
\sigma_{\varphi^{(i)}} \triangleq \sqrt{u_{\varphi^{(i)}}^{\mathrm{T}} P_{p}^{(i)} u_{\varphi^{(i)}}} .
$$

Here, $P_{p}^{(i)}$ is the position covariance of the $i$ th component and $u_{\varphi^{(i)}}$ is the unit vector orthogonal to the range direction from the $i$ th component to the sensor.

- The constant term $\sigma_{s}$ is used to scale the bearing standard deviation.

Intuitively, the operation of (35) is to reduce the nominal probability of detection at a point. The reduction depends on the weights, means and standard deviations of the components of the last estimated PHD. Reductions are only performed for the components that have smaller range values than the range of the point, and the angular proximity of the point and the components is taken into account through the exponential function in (35).

\section{ACKNOWLEDGMENT}

The authors would like to thank André Carvalho Bittencourt of Linköping University for the help with the acquisition of the data used in the experiments and Professor Fredrik Gustafsson of Linköping University for his valuable comments on the manuscript.

\section{REFERENCES}

[1] K. Gilholm and D. Salmond, "Spatial distribution model for tracking extended objects," IEE Proceedings Radar, Sonar and Navigation, vol. 152, no. 5, pp. 364-371, Oct. 2005.

[2] K. Gilholm, S. Godsill, S. Maskell, and D. Salmond, "Poisson models for extended target and group tracking," in Proceedings of Signal and Data Processing of Small Targets, vol. 5913. San Diego, CA, USA: SPIE, Aug. 2005, pp. 230-241.

[3] Y. Boers, H. Driessen, J. Torstensson, M. Trieb, R. Karlsson, and F. Gustafsson, "A track before detect algorithm for tracking extended targets," IEE Proceedings Radar, Sonar and Navigation, vol. 153, no. 4 , pp. 345-351, Aug. 2006.

[4] M. Baum and U. D. Hanebeck, "Random hypersurface models for extended object tracking," in IEEE International Symposium on Signal Processing and Information Technology (ISSPIT), Ajman, United Arab Emirates, Dec. 2009, pp. 178-183.

[5] M. Baum, B. Noack, and U. D. Hanebeck, "Extended Object and Group Tracking with Elliptic Random Hypersurface Models," in Proceedings of the International Conference on Information Fusion, Edinburgh, UK, Jul. 2010.

[6] M. Baum and U. D. Hanebeck, "Shape Tracking of Extended Objects and Group Targets with Star-Convex RHMs," in Proceedings of the International Conference on Information Fusion, Chicago, IL, USA, Jul. 2011, pp. 338-345.

[7] J. W. Koch, "Bayesian approach to extended object and cluster tracking using random matrices," IEEE Transactions on Aerospace and Electronic Systems, vol. 44, no. 3, pp. 1042-1059, Jul. 2008.

[8] J. W. Koch and M. Feldmann, "Cluster tracking under kinematical constraints using random matrices," Robotics and Autonomous Systems, vol. 57, no. 3, pp. 296-309, Mar. 2009.

[9] M. Feldmann, D. Fränken, and J. W. Koch, "Tracking of extended objects and group targets using random matrices," IEEE Transactions on Signal Processing, vol. 59, no. 4, pp. 1409-1420, Apr. 2011. 
[10] W. Wieneke and J. W. Koch, "Probabilistic tracking of multiple extended targets using random matrices," in Proceedings of SPIE Signal and Data Processing of Small Targets, Orlando, FL, USA, Apr. 2010.

[11] M. Baum, M. Feldmann, D. Fränken, U. D. Hanebeck, and J. W. Koch, "Extended object and group tracking: A comparison of random matrices and random hypersurface models," in Proceedings of the IEEE ISIF Workshop on Sensor Data Fusion: Trends, Solutions, Applications (SDF), Leipzig, Germany, Oct. 2010.

[12] D. J. Salmond and M. C. Parr, "Track maintenance using measurements of target extent," IEE Proceedings - Radar, Sonar and Navigation, vol. 150, no. 6, pp. 389-395, Dec. 2003.

[13] K. Granström, C. Lundquist, and U. Orguner, "Tracking Rectangular and Elliptical Extended Targets Using Laser Measurements," in Proceedings of the International Conference on Information Fusion, Chicago, IL, USA, Jul. 2011, pp. 592-599.

[14] C. Lundquist, K. Granström, and U. Orguner, "Estimating the Shape of Targets with a PHD Filter," in Proceedings of the International Conference on Information Fusion, Chicago, IL, USA, Jul. 2011, pp. $49-56$.

[15] H. Zhu, C. Han, and C. Li, "An extended target tracking method with random finite set observations," in Proceedings of the International Conference on Information Fusion, Chicago, IL, USA, Jul. 2011, pp. 73-78.

[16] R. Mahler, Statistical Multisource-Multitarget Information Fusion. Norwood, MA, USA: Artech House, 2007.

[17] _ - "Multitarget Bayes filtering via first-order multi target moments," IEEE Transactions on Aerospace and Electronic Systems, vol. 39, no. 4, pp. 1152-1178, Oct. 2003.

[18] B.-N. Vo and W.-K. Ma, "The Gaussian mixture probability hypothesis density filter," IEEE Transactions on Signal Processing, vol. 54, no. 11, pp. 4091-4104, Nov. 2006.

[19] R. Mahler, "PHD filters for nonstandard targets, I: Extended targets," in Proceedings of the International Conference on Information Fusion, Seattle, WA, USA, Jul. 2009, pp. 915-921.

[20] K. Granström, C. Lundquist, and U. Orguner, "A Gaussian mixture PHD filter for extended target tracking," in Proceedings of the International Conference on Information Fusion, Edinburgh, UK, Jul. 2010.

[21] K. Panta, D. Clark, and B.-N. Vo, "Data association and track management for the Gaussian mixture probability hypothesis density filter," IEEE Transactions on Aerospace and Electronic Systems, vol. 45, no. 3 , pp. 1003-1016, Jul. 2009.

[22] D. E. Clark, K. Panta, and B.-N. Vo, "The GM-PHD Filter Multiple Target Tracker," in Proceedings of the International Conference on Information Fusion, Florence, Italy, Jul. 2006.

[23] M. Ulmke, O. Erdinc, and P. Willett, "Gaussian Mixture Cardinalized PHD Filter for Ground Moving Target Tracking," in Proceedings of the International Conference on Information Fusion, Quebec City, Canada, Jul. 2007, pp. 1-8.

[24] G.-C. Rota, "The number of partitions of a set," The American Mathematical Monthly, vol. 71, no. 5, pp. 498-504, May 1964.

[25] C. M. Bishop, Pattern recognition and machine learning. New York, USA: Springer, 2006.

[26] T. Hastie, R. Tibshirani, and J. H. Friedman, The elements of statistical learning : Data mining, inference, and prediction, 2nd ed. New York, NY, USA: Springer, 2009.

[27] D. Arthur and S. Vassilvitskii, "k-means++: The Advantages of Careful Seeding," in Proceedings of the ACM-SIAM symposium on Discrete algorithms, Philadelphi, PA, USA, Jan. 2007, pp. 1027-1035.

[28] R. Ostrovsky, Y. Rabani, L. J. Schulman, and C. Swamy, "The Effectiveness of Lloyd-Type Methods for the k-Means Problem," in Proceedings of the IEEE Symposium on Foundations of Computer Science (FOCS), Berkeley, CA, USA, Oct. 2006, pp. 165-174.

[29] L. Sorber, "k-means++," 2011, accessed 30-August2011. [Online]. Available: [http://www.mathworks.com/matlabcentral/ fileexchange/28804-k-means++]

[30] X. Rong Li and V. Jilkov, "Survey of maneuvering target tracking: Part I. Dynamic models," IEEE Transactions on Aerospace and Electronic Systems, vol. 39, no. 4, pp. 1333-1364, Oct. 2003.

[31] D. Schuhmacher, B.-T. Vo, and B.-N. Vo, "A consistent metric for performance evaluation of multi-object filters," IEEE Transactions on Signal Processing, vol. 56, no. 8, pp. 3447-3457, Aug. 2008.

[32] U. Orguner, C. Lundquist, and K. Granström, "Extended Target Tracking with a Cardinalized Probability Hypothesis Density Filter," in Proceedings of the International Conference on Information Fusion, Chicago, IL, USA, Jul. 2011, pp. 65-72.
[33] O. Erdinc, P. Willett, and Y. Bar-Shalom, "The bin-occupancy filter and its connection to the PHD filters," IEEE Transactions on Signal Processing, vol. 57, no. 11, pp. 4232 -4246, Nov. 2009.

[34] R. Mahler, "PHD filters of higher order in target number," IEEE Transactions on Aerospace and Electronic Systems, vol. 43, no. 4, pp. 1523-1543, Oct. 2007.

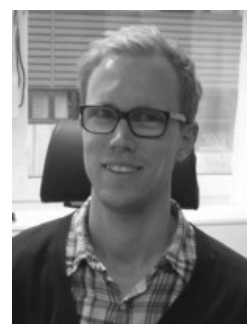

Karl Granström received the M.Sc. degree in applied physics and electrical engineering in 2008, and the Tech.Lic. degree in automatic controlin 2011, both from Linköping University, Linköping, Sweden. At present he is working towards a Ph.D. degree at the Division of Automatic Control at the Departmend of Electrical Engineering, Linköping University.

His research interests include sensor fusion, target tracking, mapping and localisation, especially for mobile robots.

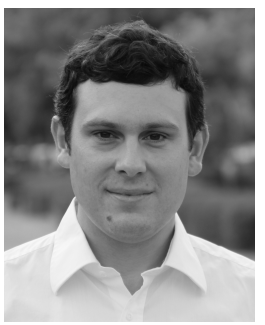

Christian Lundquist received the M.Sc. degree in automation and mechatronics engineering from Chalmers University of Technology, Gothenburg, Sweden, in 2003. He is currently working towards the Ph.D. degree at the Division of Automatic Control in the Department of Electrical Engineering of Linköping University, Linköping, Sweden.

Between 2004 and 2007, he worked with active steering systems at ZF Lenksysteme $\mathrm{GmbH}$, Germany. His research interests include sensor fusion and target tracking for automotive applications.

$\mathrm{He}$ is CEO and co-founder of the company SenionLab, developing navigation and positioning solutions for indoor usage.

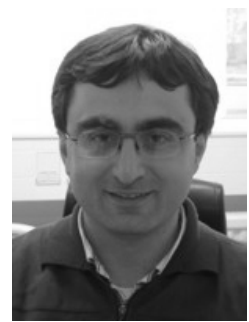

Umut Orguner is an Assistant Professor at the Division of Automatic Control at the Department of Electrical Engineering, Linköping University. He held a postdoctoral position at the same department between 2007 and 2009. He got his B.S., M.S. and $\mathrm{Ph} . \mathrm{D}$. degrees all in Electrical Engineering from Department of Electrical \& Electronics Engineering, Middle East Technical University, Ankara, Turkey in 1999, 2002 and 2006 respectively. His research interests include estimation theory, multiple-model estimation, target tracking, and information fusion. 